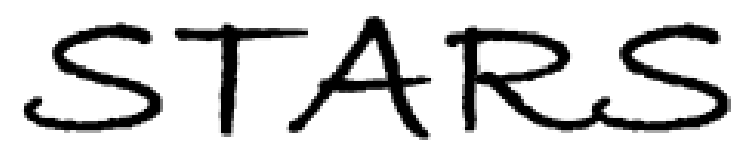

University of Central Florida

STARS

$1-1-2015$

\title{
Electromagnetic field correlations in three-dimensional speckles
}

Aristide Dogariu

University of Central Florida

Remi Carminati

University of Central Florida

Find similar works at: https://stars.library.ucf.edu/facultybib2010

University of Central Florida Libraries http://library.ucf.edu

This Review is brought to you for free and open access by the Faculty Bibliography at STARS. It has been accepted for inclusion in Faculty Bibliography 2010 s by an authorized administrator of STARS. For more information, please contact STARS@ucf.edu.

\section{Recommended Citation}

Dogariu, Aristide and Carminati, Remi, "Electromagnetic field correlations in three-dimensional speckles" (2015). Faculty Bibliography 2010s. 6509.

https://stars.library.ucf.edu/facultybib2010/6509

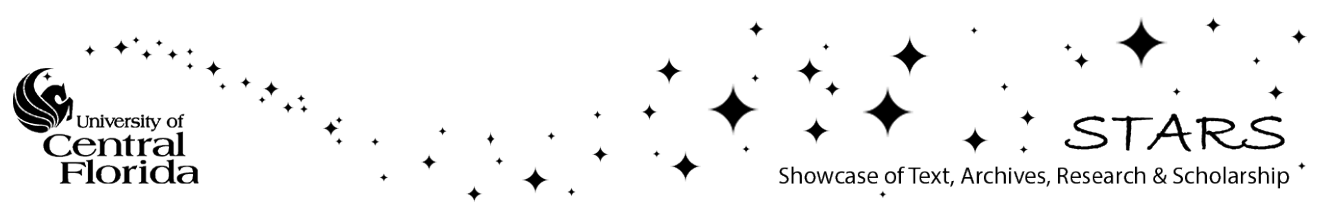




\title{
Electromagnetic field correlations in three-dimensional speckles
}

\author{
Aristide Dogariu $^{\mathrm{a}, *}$, Rémi Carminati ${ }^{\mathrm{b}}$ \\ ${ }^{a}$ CREOL, College of Optics and Photonics, University of Central Florida, Orlando, FL 32816-2700, USA \\ ${ }^{\mathrm{b}}$ ESPCI ParisTech, PSL Research University, CNRS, Institut Langevin, 1 rue Jussieu, F-75005, Paris, France
}

\section{A R T I C L E I N F O}

\section{Article history:}

Accepted 17 November 2014

Available online 25 November 2014

editor: G.I. Stegeman

\section{Keywords:}

Random fields

Speckle

Coherence

Polarization

\begin{abstract}
A B S T R A C T
We describe recent developments in the characterization of three-dimensional speckle fields produced by scattering of electromagnetic waves. In many practical situations the description of such fields requires approaches going beyond the Gaussian statistics approximation. Quantitative measures of spatial coherence and polarization can be defined from the field-field correlation matrix, known as the cross-spectral density matrix in coherence theory. The complex degree of mutual polarization provides a measure of the similarity between polarization states at two different points. The degree of spatial coherence describes spatial coherence and averages out the polarization properties. We discuss their behavior in speckle fields produced by multiple scattering in disordered materials. A number of non-universal properties arise, that are related to the internal microscopic structure of the scattering medium. Non-universality affects observables quantities, such as spatial correlations in speckle patterns measured in the near field of the medium surface, statistics of the local density of states or the depolarization of the exciting electromagnetic field due to scattering. Specific microscopic scales are necessary to describe the non-universal behaviors, that characterize the scale-dependent morphology of the scattering medium.
\end{abstract}

(C) 2015 Published by Elsevier B.V.

\section{Contents}

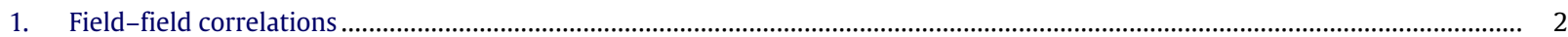

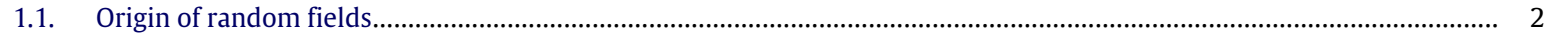

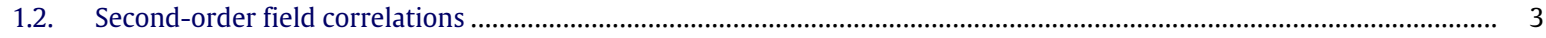

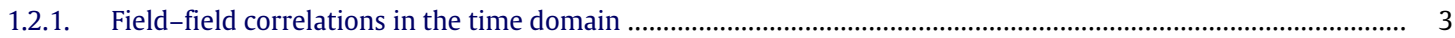

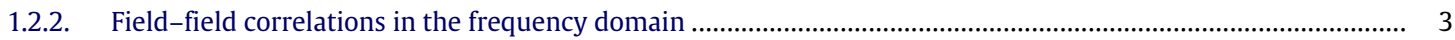

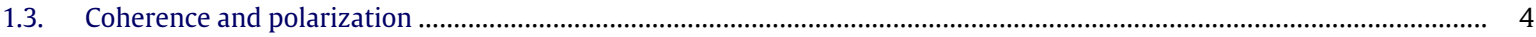

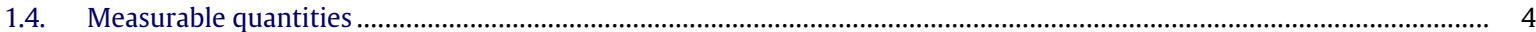

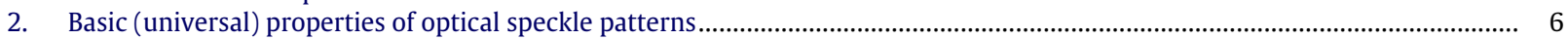

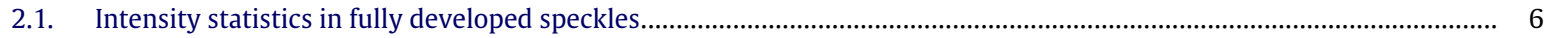

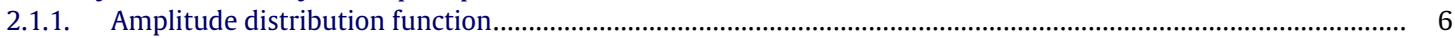

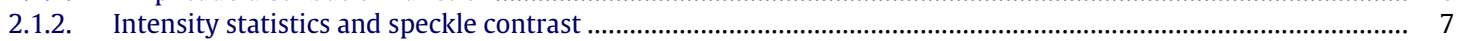

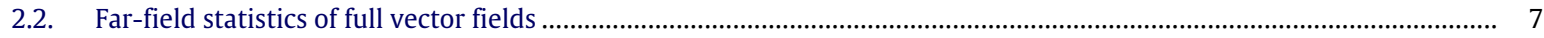

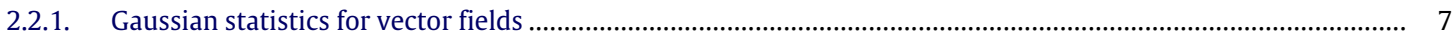

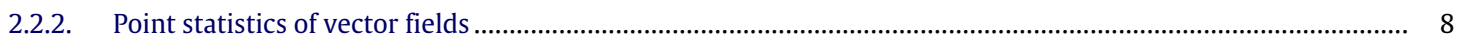

\footnotetext{
* Corresponding author.

E-mail addresses: aristide.dogariu@creol.ucf.edu (A. Dogariu), remi.carminati@espci.fr (R. Carminati).
} 
2.2.3. Two-point properties of vector fields

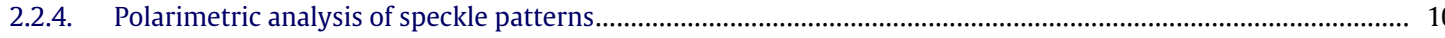

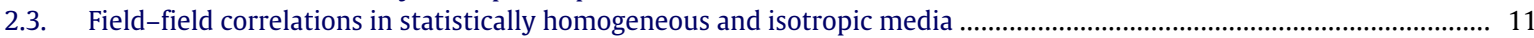

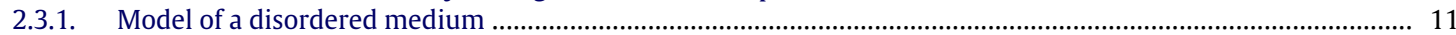

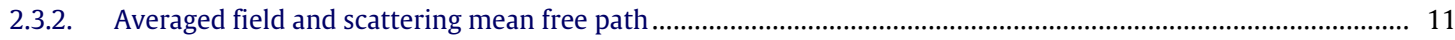

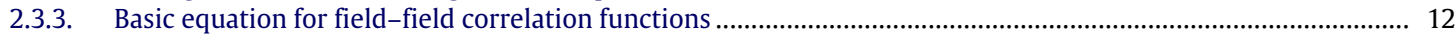

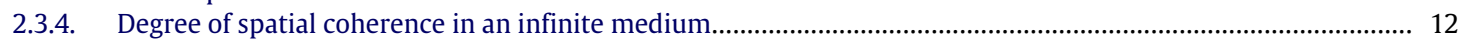

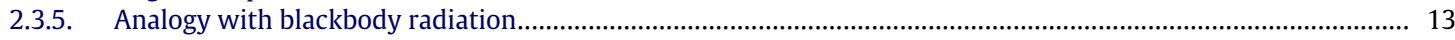

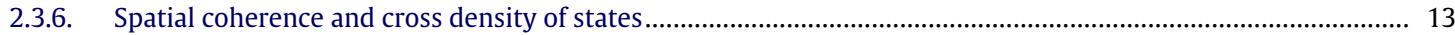

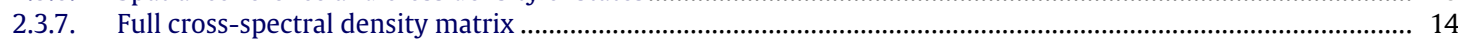

2.3.8. Transport models for spatial coherence and polarization .......................................................................... 15

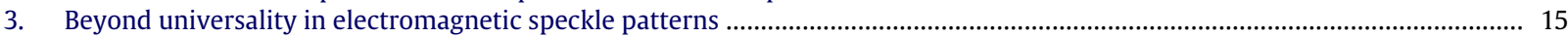

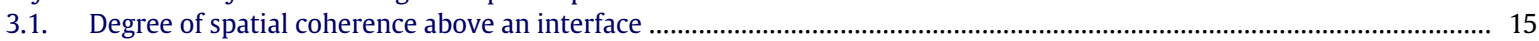

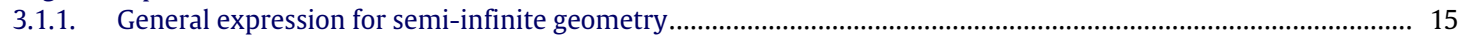

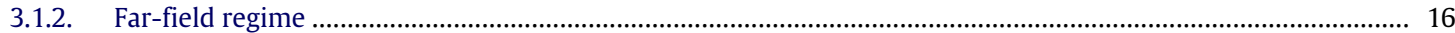

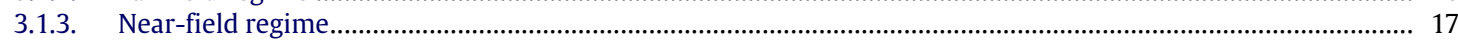

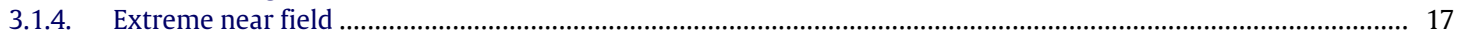

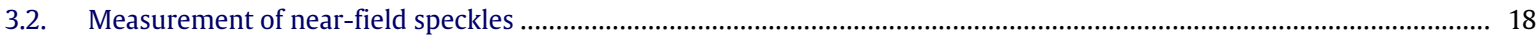

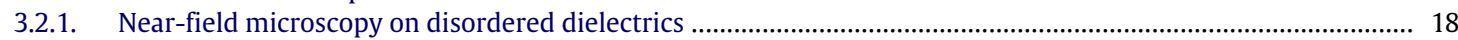

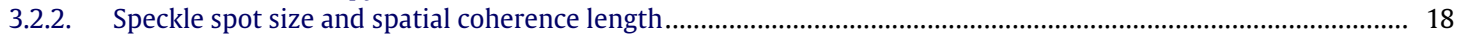

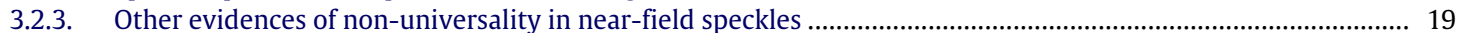

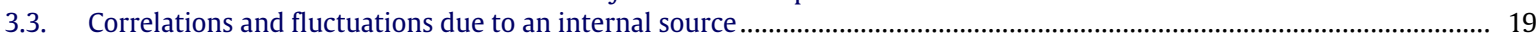

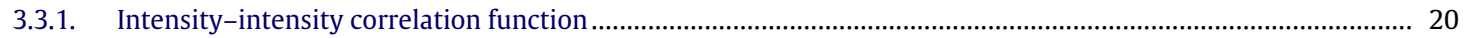

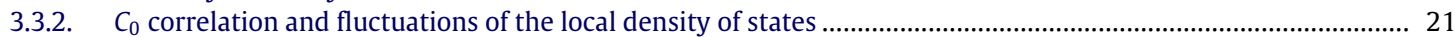

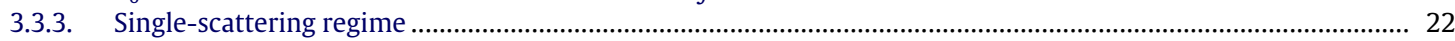

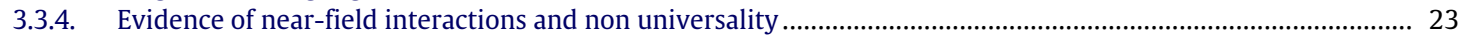

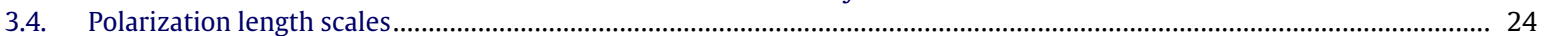

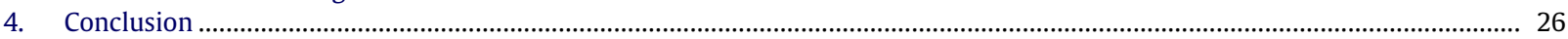

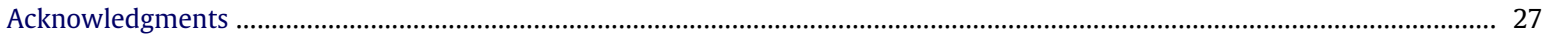

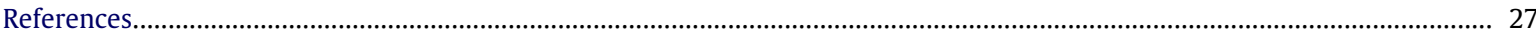

\section{Field-field correlations}

\subsection{Origin of random fields}

The description of light-matter interaction in a deterministic framework becomes severely limited when real situations in physics or engineering need to be addressed. Temporal fluctuations in light emission from primary sources are inherent to the process of spontaneous emission. Even the light emitted by a laser is subject to fluctuations due to spontaneous emission noise. Along the years, the need for a characterization and a classification of the statistical behavior of optical fields, accounting for the fundamentally statistical aspect of light emission, has motivated the development of statistical optics and coherence theory [1,2]. At a given point $\mathbf{r}$, the field amplitude $E(\mathbf{r}, t)$ fluctuates in time. In coherence theory, the amplitude $E(\mathbf{r}, t)$ is considered as a random variable resulting from a stochastic process that generates a statistical ensemble of realizations of the field. In classical optics, second-order field-field correlations at one point and two different times describe temporal coherence, while equal time field-field correlations at two different points describe spatial coherence. These second-order statistical quantities drive the contrast in two-wave interference experiments as in the Michelson interferometer or in Young's two-slit experiment. Since light is an electromagnetic wave, field-field correlations of different vector components of the electric field describe the polarization properties [3-5]. Indeed, polarization is a statistical concept that has to be handled on equal footing as temporal and spatial coherence [6].

Light-matter interaction in heterogeneous materials also produces spatial fluctuations of the field that are commonly referred to as speckle [7]. If the material has some internal dynamics (as, for example, in a colloidal suspension of particles), temporal fluctuations can also be induced forming a so-called dynamic speckle pattern [8]. A description of speckle fluctuations in terms of deterministic field amplitudes is in practice hopeless due to the impossibility to determine precisely the internal microscopic structure of the scattering medium. But more fundamentally it turns out to be even useless since many macroscopic quantities only depend on some averaged parameters that describe statistical features of the medium, such as the number density of scatterers or the scattering mean free path. For example, ten identical glasses filled with milk taken from the same bottle exhibit the same global transmittance or reflectance, although the microscopic structures of the milk (positions of the colloidal particles) in each glass are not the same. Therefore it seems appropriate to handle the problem of the prediction of the reflectance or transmittance of a glass of milk starting from the beginning with a statistical approach in which the light field is considered as a random variable.

In summary, whether the source of fluctuations is the light emission process itself or the scattering of light by disordered materials, the description of electromagnetic speckles is based on a statistical approach similar to that used more generally in statistical physics. The field is considered to be a random variable, generated by an underlying stochastic process. The 
statistical properties of the stochastic process can be inferred from general assumptions, and are directly connected to observables associated to the coherence and polarization properties of the field.

\subsection{Second-order field correlations}

It is now well understood that, in the case of propagating optical fields, the second-order correlations between orthogonal components of the fluctuating electric field vector provide the entire information about the field [9]. In a scalar model, the electromagnetic field at a position vector $\mathbf{r}$ and a time $t$ can be represented by the real variable $E^{(r)}(\mathbf{r}, t)$. In any realistic situation where the light originates from a thermal source or a laser, $E^{(r)}(\mathbf{r}, t)$ fluctuates as a function of time and, therefore, only a statistical approach can be considered in which $E^{(r)}(\mathbf{r}, t)$ is considered as a random variable. A statistical approach in the frequency domain is also of interest, either to characterize the spectral properties of the field $E^{(r)}(\mathbf{r}, t)$, or to characterize a monochromatic field that fluctuates in space, as in a speckle pattern produced by scattering of a stabilized laser beam. In the spectral domain the field is represented by a complex random variable $E(\mathbf{r}, \omega)$.

\subsubsection{Field-field correlations in the time domain}

In the time domain, it is mathematically convenient to use the analytic signal $E(\mathbf{r}, t)$ associated with the real field variable $E^{(r)}(\mathbf{r}, t)$. One can regard $E(\mathbf{r}, t)$ as a particular member of the statistical ensemble $\{E(\mathbf{r}, t)\}$ composed of all the possible field realizations. Since the oscillations of an optical field are too fast to be recorded by any detector, the measured quantity is often proportional to the intensity, which depends on the second-order correlation of the field at two space-time points. The correlation between the fluctuations of the field $E\left(\mathbf{r}_{1}, t_{1}\right)$ at position $\mathbf{r}_{1}$ and time $t_{1}$, and the fluctuations of the field $E\left(\mathbf{r}_{2}, t_{2}\right)$ at position $\mathbf{r}_{2}$ and time $t_{2}$ is evaluated by the cross-correlation function defined as

$$
\Gamma\left(\mathbf{r}_{1}, \mathbf{r}_{2}, t_{1}, t_{2}\right)=\left\langle E^{*}\left(\mathbf{r}_{1}, t_{1}\right) E\left(\mathbf{r}_{2}, t_{2}\right)\right\rangle
$$

where $\langle\cdot\rangle$ denotes the ensemble average over the realizations of the field and the superscript $*$ stands for complex conjugation. In practice the field is assumed to be ergodic and therefore the ensemble average is equivalent to a time average. In most applications the field can be considered to be stationary, at least in the wide sense, meaning that the random process is independent of the origin of time (at least its first and second moments). In this case the cross-correlation function depends only on the time difference $\tau=t_{1}-t_{2}$. Consequently, $\Gamma\left(\mathbf{r}_{1}, \mathbf{r}_{2}, t_{1}, t_{2}\right)$ can be replaced by the mutual coherence function [2]

$$
\Gamma\left(\mathbf{r}_{1}, \mathbf{r}_{2}, \tau\right)=\left\langle E^{*}\left(\mathbf{r}_{1}, t\right) E\left(\mathbf{r}_{2}, t+\tau\right)\right\rangle .
$$

Note that $\Gamma(\mathbf{r}, \mathbf{r}, 0)$ represents the average intensity at point $\mathbf{r}$, defined as $\langle I(\mathbf{r})\rangle=\left\langle|E(\mathbf{r}, t)|^{2}\right\rangle$.

\subsubsection{Field-field correlations in the frequency domain}

A field that fluctuates in time cannot be considered monochromatic. The Fourier transform of the mutual coherence function defines the cross-spectral density function

$$
W\left(\mathbf{r}_{1}, \mathbf{r}_{2}, \omega\right)=\int_{-\infty}^{+\infty} \Gamma\left(\mathbf{r}_{1}, \mathbf{r}_{2}, \tau\right) \exp (i \omega \tau) d \tau .
$$

This relation can be understood as a generalized form of the Wiener-Khintchine theorem. Indeed, for $\mathbf{r}_{1}=\mathbf{r}_{2}=\mathbf{r}, W(\mathbf{r}, \mathbf{r}, \omega)$ is the power spectral density of the field which is the Fourier transform of the time-domain field correlation function $\Gamma(\mathbf{r}, \mathbf{r}, \tau)=\left\langle E^{*}(\mathbf{r}, t) E(\mathbf{r}, t+\tau)\right\rangle$.

For statistically stationary field fluctuations, the time-domain Fourier transform of the field $E(\mathbf{r}, t)$ is not defined and the cross-spectral density function cannot be defined as a field-field correlation of the Fourier transform of the field. Nevertheless, it has been shown that $W\left(\mathbf{r}_{1}, \mathbf{r}_{2}, \omega\right)$ represents the correlation between two monochromatic members of a suitably constructed ensemble $\{E(\mathbf{r}, \omega)\}$ composed of all the monochromatic realizations of the field [2]. The cross-spectral density function then takes the form

$$
W\left(\mathbf{r}_{1}, \mathbf{r}_{2}, \omega\right)=\left\langle E^{*}\left(\mathbf{r}_{1}, \omega\right) E\left(\mathbf{r}_{2}, \omega\right)\right\rangle
$$

where $\langle\cdot\rangle$ denotes the ensemble average over the monochromatic realizations of the field (which is not a direct measurable quantity). Such a representation is very effective when dealing with second-order correlation in the spectral domain, and we shall use it extensively in this review. When the field is really monochromatic and only fluctuates in space (as in a speckle pattern created under monochromatic illumination), then the spectral representation of the cross-spectral density in Eq. (4) involves the real measurable field.

Because the cross-spectral density depends on the spatial distribution of the intensity, it is useful to normalize $W\left(\mathbf{r}_{1}, \mathbf{r}_{2}, \omega\right)$ by defining the so-called spectral degree of coherence as

$$
\mu\left(\mathbf{r}_{1}, \mathbf{r}_{2}, \omega\right)=\frac{W\left(\mathbf{r}_{1}, \mathbf{r}_{2}, \omega\right)}{\sqrt{\left\langle I\left(\mathbf{r}_{1}, \omega\right)\right\rangle\left\langle I\left(\mathbf{r}_{2}, \omega\right)\right\rangle}}
$$

where $\langle I(\mathbf{r}, \omega)\rangle=W(\mathbf{r}, \mathbf{r}, \omega)$. The spectral degree of coherence is a measure of spatial coherence in a scalar field. 


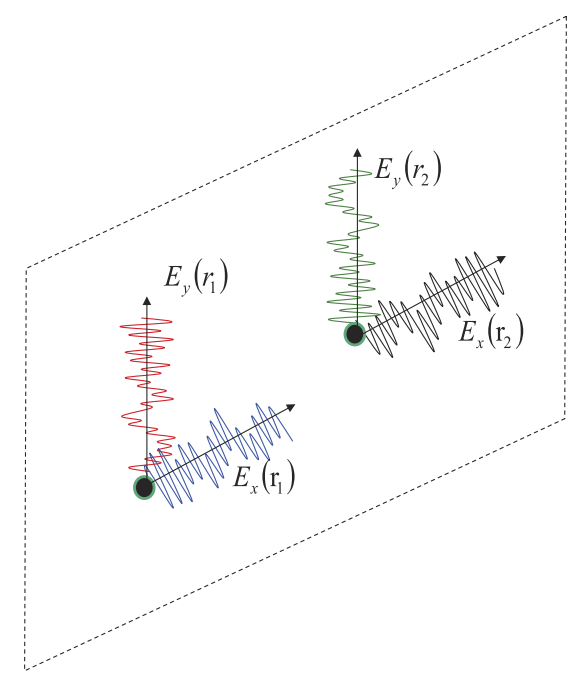

Fig. 1. The variation of electric field vector of light is a random process in both time and space. Second-order field correlations are sufficient to describe phenomena such as radiation of light, propagation, diffraction and interference. When describing the physical situation of transversal fields, one inspects and quantifies properties of four different temporal fluctuations.

\subsection{Coherence and polarization}

Electromagnetic waves are vector waves and characterizing the degree of coherence and polarization requires a formalism going beyond the scalar model. There are many situations in which the electromagnetic field propagates close to a given direction and can be regarded as beam-like. It follows that the electric field mainly lays in a plane transverse to the direction of propagation. The correlation between two orthogonal components of the electric field characterizes the polarization of the beam [6]. In this situation, the second-order statistical properties of the beam in the spectral domain are described by the elements of the cross-spectral density matrix $\mathbf{W}[2]$

$$
W_{j k}\left(\mathbf{r}_{1}, \mathbf{r}_{2}, \omega\right)=\left\langle E_{j}^{*}\left(\mathbf{r}_{1}, \omega\right) E_{k}\left(\mathbf{r}_{2}, \omega\right)\right\rangle
$$

where $j$ and $k$ are two orthogonal directions in a plane transverse to the beam axis.

The two-point correlation functions are usually associated to the spatial coherence properties of the electromagnetic field. However, given the joint probability distribution of the different components of the complex field, it is possible to calculate the statistical distribution of any measurable quantity, including one-point functions. The latter are often introduced in the description of polarization. This is usually done in terms of the normalized Stokes vector $(q, u, v)$ whose elements are defined by

$$
q=\frac{\left\langle E_{x} E_{x}^{*}-E_{y} E_{y}^{*}\right\rangle}{\left\langle E_{x} E_{x}^{*}+E_{y} E_{y}^{*}\right\rangle} \quad u=\frac{\left\langle E_{x} E_{y}^{*}+E_{y} E_{x}^{*}\right\rangle}{\left\langle E_{x} E_{x}^{*}+E_{y} E_{y}^{*}\right\rangle} \quad v=\frac{i\left\langle E_{x} E_{x}^{*}-E_{y} E_{y}^{*}\right\rangle}{\left\langle E_{x} E_{x}^{*}+E_{y} E_{y}^{*}\right\rangle}
$$

where $E_{x, y}=E_{x, y}(\mathbf{r}, \omega)$ are the complex electric field in two orthogonal directions [3]. It is important to realize that, because this representation is in terms of intensity distributions along two orthogonal directions, the information about absolute phase is lost. In fact, having access only to the distribution of the states of polarization, even the intensity information is lost and the only surviving property is the joint probability distribution of states of polarization $p(q, u, v)=\int p(I, q, u, v) d I$. For visualization purposes, this distribution is usually examined in spherical coordinates $(r, \theta, \phi)$ on the Poincaré sphere with $q=r \cos \phi, u=r \sin \phi \cos \theta, v=r \sin \phi \sin \theta$ and $p(r, \theta, \phi)=r^{2} \sin \phi p(q, u, v)$ (the assumption of pure states of polarization forces $r=1$ ). This representation allows one to examine statistical ensembles with different degrees of polarization. An example of a representation on the Poincaré sphere is shown in Fig. 4.

\subsection{Measurable quantities}

For electromagnetic fields transverse to the direction of propagation, the fluctuations of the electric field at any positions $\mathbf{r}$ can be decomposed along two orthogonal directions schematically depicted in Fig. 1. When inspecting the second-order statistics, the field properties are then described by all the possible correlations $\left\langle E_{j}^{*} E_{k}\right\rangle$ between these random functions. This operation can be performed at one single spatial point $\mathbf{r}$ and this constitutes the essence of so-called Stokes polarimetry. One can also examine mutual properties by evaluating the cross-correlations between field fluctuations at two different points $\mathbf{r}_{1}$ and $\mathbf{r}_{2}$ (using some sort of interferometric approach similar to Young's two-source experiment for instance).

Mathematically, the description of the second-order correlation properties of a beam-like fluctuating electromagnetic field $\mathbf{E}(\mathbf{r}, \omega)$ involves the complete set of complex-valued field-field correlation functions $W_{j k}\left(\mathbf{r}_{1}, \mathbf{r}_{2}, \omega\right)$ included in the 


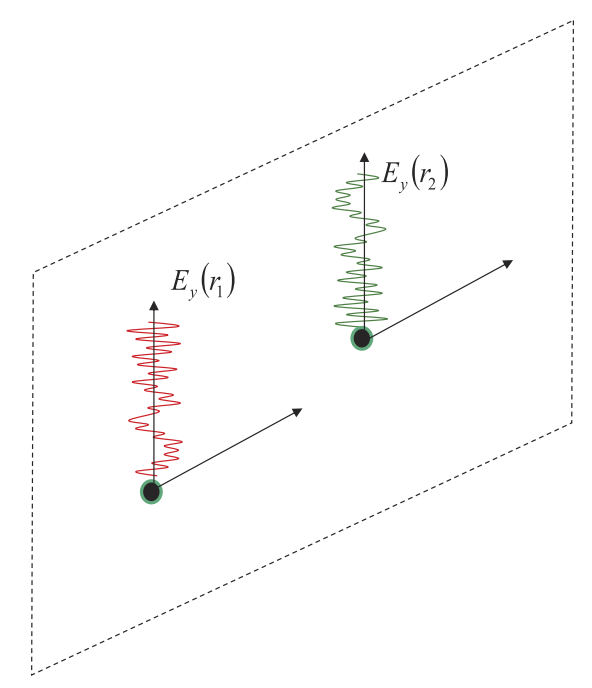

Fig. 2. Gauging the degree of coherence requires the measurement of three different elements of $W$ : the intensities $\left\langle E_{y}^{*}\left(\mathbf{r}_{1}\right) E_{y}\left(\mathbf{r}_{1}\right)\right\rangle$ and $\left\langle E_{y}^{*}\left(\mathbf{r}_{2}\right) E_{y}\left(\mathbf{r}_{2}\right)\right\rangle$, and the cross-correlation function $\left\langle E_{y}^{*}\left(\mathbf{r}_{1}\right) E_{y}\left(\mathbf{r}_{2}\right)\right\rangle$.

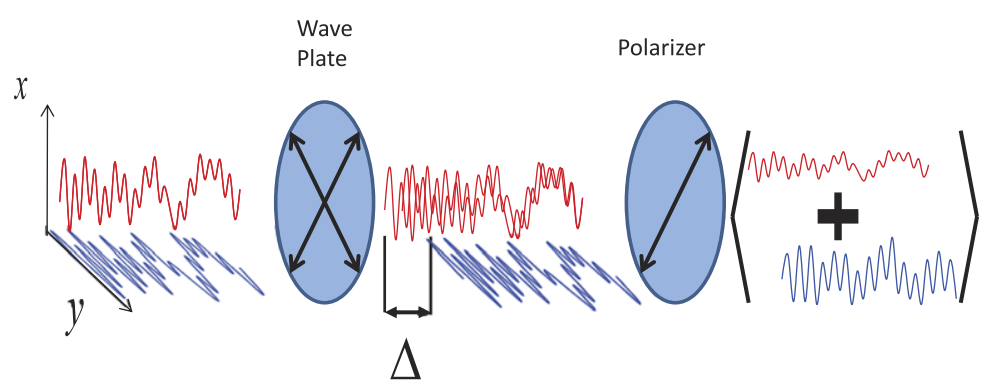

Fig. 3. The interferometric measurement of polarization where the polarimeter is represented as a common path interferometer.

cross-spectral density matrix $\mathbf{W}$. It is essential to realize that the entire information that can be described through secondorder field correlations is included in $\mathbf{W}$. To access it, one needs at least sixteen independent measurements.

It is also important to note that, for optical fields at least, knowledge of the field means in fact knowledge of time averaged quantities. The measurable characteristics discussed so far, intensity, Stokes vector, mutual intensity, or cross-spectral density are all ensemble averaged quantities of certain second-order field correlations.

Another significant observation is that, sometimes, physical measurements are not intended to provide the individual elements of the cross-spectral density matrix $\mathbf{W}$. Instead, they focus on lossy compressions of information expressed through degrees such as degree of coherence, polarization, etc. Take for instance the degree of coherence. A scalar interpretation treats it as a phase similarity, statistical similarity, or ability for the field at two points to interfere. A vector theory on the other hand does not have a unique answer and more specific questions need to be asked. This interplay has led to some semantic arguments concerning second-order coherence and polarization information [10]. For instance, orthogonally polarized, fully correlated fields do not produce interference, and one may argue that they are incoherent. However, a simple and reversible operation can alter them in such a way that maximal visibility is obtained and, then, the fields may be considered coherent.

One could certainly debate as to whether coherence properties should be polarization independent [11], free of any point property [12], or if the separation between polarization and coherence can even be made [13]. However, more relevant questions relate to what can actually be measured about a random electromagnetic field and, most importantly, to what does one need to know about the field in a particular situation. The physically significant inquiry concerns the maximum visibility which can be obtained when the field is modified in certain way by altering the intensity, the state of polarization, relative phases, etc.

In this context one should be aware that all optical measurements are, in essence, interferometric. For example, to infer the two-point coherence one needs four visibility measurements to determine the maxima and minima of intensities as well as their positions, as illustrated in Fig. 2. Importantly, in order to determine $\left\langle E_{y}^{*}\left(\mathbf{r}_{1}\right) E_{y}\left(\mathbf{r}_{2}\right)\right\rangle$ one also requires the single point measurements at $\mathbf{r}_{1}$ and $\mathbf{r}_{2}$.

Polarization is also assessed interferometrically as illustrated in Fig. 3. A typical polarization measurement uses a phase plate to delay the electric field fluctuations along two orthogonal directions, a subsequent polarizer adjusts the relative magnitude of these field components, and an intensity detector integrates in time their superposition creating the neces- 
sary ensemble average. A typical polarization measurement requires four different interferometric experiments in which the delay $\Delta$ is adjusted by rotating the waveplate, and the relative magnitudes of the fields are changed by adjusting the orientation of the polarizer [3]. A two-point optical situation as illustrated in Fig. 1 is fully described by the two-point correlation functions forming the cross-spectral density matrix $\mathbf{W}$ which is defined, in general, by sixteen different parameters. Thus, a complete characterization of the field second-order properties requires sixteen independent measurements. Some of the elements of $\mathbf{W}$ contribute to what is usually called spatial coherence of the field and some other to what is commonly regarded as polarization. Of course, in an effort to synthesize the measurable information one could further combine some or even all elements of $\mathbf{W}$ and obtain different other parameters. Nevertheless, this cannot provide additional facts about the field characteristics. The only direct observables in optical experiments are the second-order correlation functions, i.e. exactly the elements $W_{j k}$, as pointed out by Wolf sixty years ago in a ground breaking work [14].

\section{Basic (universal) properties of optical speckle patterns}

When a heterogeneous disordered medium is illuminated by a monochromatic electromagnetic wave at a frequency $\omega$, a strongly fluctuating intensity pattern is produced, that is known as a speckle pattern. The first reported observation of a speckle pattern produced by coherent laser light seems to be due to Langmuir, in 1963, shortly after the discovery of the laser [15]. In a so-called fully developed speckle pattern, the field at any point is the superposition of a large number of partial waves that are statistically independent in both amplitude and phase. By virtue of the central limit theorem, the field exhibits Gaussian statistics. In a fully developed speckle pattern, the intensity statistics and the field-field correlation functions that characterize spatial coherence and polarization exhibit general features that we review in this section.

\subsection{Intensity statistics in fully developed speckles}

In this section we briefly recall the concept of fully developed speckle, and the derivation of intensity statistics. We describe the scattering medium using a statistical ensemble of realizations of disorder. The field at a given point $\mathbf{r}$ of the speckle pattern is of the form $\mathbf{E}(\mathbf{r}, t)=\operatorname{Re}[\mathbf{E}(\mathbf{r}) \exp (-i \omega t)]$, where $\omega=2 \pi c / \lambda$ is the frequency of the incident wave and $\lambda$ the wavelength in vacuum. The complex amplitude $\mathbf{E}(\mathbf{r})$ and the intensity $I(\mathbf{r})=|\mathbf{E}(\mathbf{r})|^{2}$ are random variables. Assuming ergodicity the statistical properties of $\mathbf{E}(\mathbf{r})$ and $I(\mathbf{r})$ can be compared to that obtained in practice by using spatial averages on a given realization of a speckle pattern.

\subsubsection{Amplitude distribution function}

The statistical distribution of one component $E_{\alpha}(\mathbf{r})$ of the complex amplitude of the electric field along direction $\alpha=x$, $y, z$ can be deduced from the central limit theorem. A given component of the field, or the intensity $I_{\alpha}(\mathbf{r})=\left|E_{\alpha}(\mathbf{r})\right|^{2}$, can be measured after a polarizer or using a linearly polarized detector (dipole antenna).

The complex amplitude $E_{\alpha}(\mathbf{r})$ results from a scattering process that involves a large number of partial waves. It can be written in the form

$$
E_{\alpha}(\mathbf{r})=\sum_{n=1}^{+\infty} \sum_{\left\{\mathbf{r}_{1}, \mathbf{r}_{2} \cdots \mathbf{r}_{n}\right\}} A_{\delta_{n}}(\mathbf{r}) \exp \left[i \phi_{\delta_{n}}(\mathbf{r})\right]
$$

where $s_{n}$ is a scattering sequence involving $n$ scattering events at position $\left\{\mathbf{r}_{1}, \mathbf{r}_{2} \cdots \mathbf{r}_{n}\right\}$ (in a medium made of discrete particles, these are the locations of the $n$ particles involved in the sequence). $A_{s_{n}}(\mathbf{r})$ and $\phi_{s_{n}}(\mathbf{r})$ are the amplitude and phase of the partial wave arising from the scattering sequence $s_{n}$. We shall use the simplified notation

$$
E_{\alpha}(\mathbf{r})=\sum_{\delta} A_{\delta}(\mathbf{r}) \exp \left[i \phi_{\delta}(\mathbf{r})\right]
$$

where $s$ denotes a scattering sequence. Both $A_{s}(\mathbf{r})$ and $\phi_{s}(\mathbf{r})$ are random variables. A comprehensive study of the statistical properties of fields given by Eq. (9), denoted by random phasor sums, can be found in Refs. [1,7]. The model of fully developed speckle relies on the following assumptions:

1. The complex amplitudes resulting from two different sequences $s$ and $\delta^{\prime}$ are independent random variables.

2. For a given sequence $\delta$, the amplitude $A_{\S}(\mathbf{r})$ and the phase $\phi_{\delta}(\mathbf{r})$ are mutually uncorrelated.

3. The phase $\phi_{s}(\mathbf{r})$ is uniformly distributed on $[0,2 \pi]$.

The sum (9) contains a large number of terms, that are independent complex random variables. In these conditions, an application of the central limit theorem is possible. The real and imaginary parts of the field, $X=\operatorname{Re} E_{\alpha}(\mathbf{r})$ and $Y=\operatorname{Im} E_{\alpha}(\mathbf{r})$, read:

$$
\begin{aligned}
& X=\sum_{\delta} A_{\delta}(\mathbf{r}) \cos \phi_{\delta}(\mathbf{r}) \\
& Y=\sum_{\delta} A_{\delta}(\mathbf{r}) \sin \phi_{\delta}(\mathbf{r}) .
\end{aligned}
$$


One can deduce the average value, variance and cross-correlation of the two real random variables $X$ and $Y$ under the three hypotheses of the model of fully developed speckle. The average values are simply $\langle X\rangle=\langle Y\rangle=0$. The variance is $\sigma^{2}=\left\langle X^{2}\right\rangle$ $=\left\langle Y^{2}\right\rangle=(1 / 2) \sum_{s}\left\langle A_{s}^{2}(\mathbf{r})\right\rangle$. Moreover, it is also easy to verify that $\langle X Y\rangle=0$, showing that the real and imaginary parts of the field are uncorrelated. The central limit theorem states that, in the limit of an infinite number of terms in the summation (9), both $X$ and $Y$ are Gaussian variables with zero mean and equal variance. The joint probability of $X$ and $Y$ is also Gaussian:

$$
p(X, Y)=\frac{1}{2 \pi \sigma^{2}} \exp \left(-\frac{X^{2}+Y^{2}}{2 \sigma^{2}}\right) .
$$

From this joint distribution, the statistics of the field amplitude $\left|E_{\alpha}\right|=\sqrt{X^{2}+Y^{2}}$ is obtained through a change of variable (we drop the dependence on $\mathbf{r}$ for simplicity):

$$
\begin{aligned}
& p\left(\left|E_{\alpha}\right|\right)=\frac{\left|E_{\alpha}\right|}{\sigma^{2}} \exp \left(-\frac{\left|E_{\alpha}\right|^{2}}{2 \sigma^{2}}\right) \quad \text { for }\left|E_{\alpha}\right| \geq 0 \\
& p\left(\left|E_{\alpha}\right|\right)=0 \text { for }\left|E_{\alpha}\right|<0 .
\end{aligned}
$$

\subsubsection{Intensity statistics and speckle contrast}

The probability density of the intensity $I_{\alpha}=\left|E_{\alpha}\right|^{2}$ associated to one component of the field follows from Eq. (11) by making use of the change of variable

$$
p\left(I_{\alpha}\right)=p\left(\left|E_{\alpha}\right|=\sqrt{I_{\alpha}}\right) \frac{d\left|E_{\alpha}\right|}{d I_{\alpha}}=\frac{1}{2 \sqrt{I_{\alpha}}} p\left(\left|E_{\alpha}\right|=\sqrt{I_{\alpha}}\right) .
$$

We obtain the exponential statistics, known as the Rayleigh statistics:

$$
\begin{aligned}
& p\left(I_{\alpha}\right)=\frac{1}{\left\langle I_{\alpha}\right\rangle} \exp \left(-\frac{I_{\alpha}}{\left\langle I_{\alpha}\right\rangle}\right) \quad \text { for } I_{\alpha} \geq 0 \\
& p\left(I_{\alpha}\right)=0 \text { for } I_{\alpha}<0
\end{aligned}
$$

where $\left\langle I_{\alpha}\right\rangle=2 \sigma^{2}$ is the averaged intensity. This form of the intensity distribution is a feature of fully developed speckle patterns. It is interesting to note that the most likely value of the intensity due to one component of the field is $I_{\alpha}=0$.

The intensity fluctuations in the speckle pattern produced by the component $E_{\alpha}$ are characterized by the variance $\sigma_{I_{\alpha}}^{2}=\left\langle I_{\alpha}^{2}\right\rangle-\left\langle I_{\alpha}\right\rangle^{2}$. The second moment of the intensity is obtained from Eq. (13) using an integration by part:

$$
\left\langle I_{\alpha}^{2}\right\rangle=\int_{0}^{\infty} I_{\alpha}^{2} p\left(I_{\alpha}\right) d I_{\alpha}=2\left\langle I_{\alpha}\right\rangle^{2} .
$$

The speckle contrast is defined as the normalized standard deviation $\sigma_{I_{\alpha}} /\langle I\rangle$. Eq. (14) leads to the very simple result

$$
\frac{\sigma_{I_{\alpha}}}{\left\langle I_{\alpha}\right\rangle}=1
$$

showing that a fully developed speckle pattern exhibits large intensity fluctuations. This is another feature of the Rayleigh statistics.

\subsection{Far-field statistics of full vector fields}

Expression (13) describes the statistics of the intensity produced by one component of the electric field. It corresponds to a measurement of a linearly polarized field. Finding the statistical distribution of the full intensity, measured without reducing the field to one of its vector components, is a more complicated task. In this case, a number of measurable distributions, including intensity, phase difference, ellipticity, and states of polarization, can be derived from the statistics of random complex fields.

\subsubsection{Gaussian statistics for vector fields}

In a three-dimensional speckle, such as that observed inside a disordered medium or in close proximity of its surface, the complex amplitude of the field is the superposition of three independent complex random variables. However, when the speckle is observed in the far field as a paraxial field only two independent components need to be accounted for. In this case, it is customary to visualize the polarization statistics as a distribution of specific states mapped onto a Poincaré sphere of unit radius where pure states are distributed across its surface while partially polarized and unpolarized states lie somewhere inside the sphere [3].

The speckle seen as a whole is often considered to represent completely unpolarized light. Every point in a globally depolarized field, whether it is a point in space or time or a member of an ensemble, can be considered the result of the 


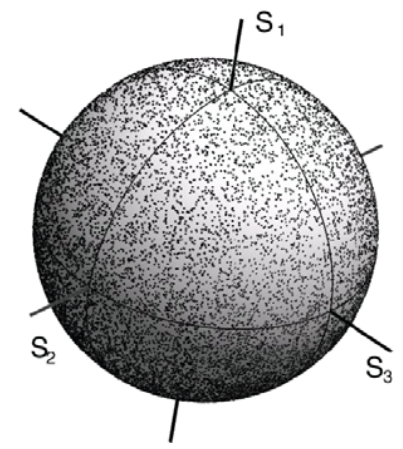

Fig. 4. A uniform random distribution of polarization states on the Poincaré sphere describes an ensemble of realizations of random electromagnetic fields which are locally fully polarized but, upon averaging, represent an unpolarized field.

summation of a large number of underlying fields. The probability distribution of these underlying fields determines the state of polarization at that particular point. It is possible that these underlying fields are locally unpolarized, in which case there is no other information beyond the intensity statistics. It is also possible that the underlying fields are locally polarized, in which case the ensemble of polarization states is represented by a specific distribution on the surface of the Poincaré sphere.

A uniform distribution of polarization states across the Poincaré sphere is invariant to any possible rotation of the coordinate axis, as well as symmetric about a specific plane and it would be representative for unpolarized radiation (see Fig. 4). Of course, the most common distribution of a complex vector field with such statistics is the independent Gaussian distributed field, characterized by the distribution function

$$
p\left(E_{x}^{\prime}, E_{x}^{\prime \prime}, E_{y}^{\prime}, E_{y}^{\prime \prime}\right)=\frac{1}{4 \pi^{2} \sigma^{4}} \exp \left[-\frac{\left(E_{x}^{\prime}\right)^{2}+\left(E_{x}^{\prime \prime}\right)^{2}+\left(E_{y}^{\prime}\right)^{2}+\left(E_{y}^{\prime \prime}\right)^{2}}{2 \sigma^{2}}\right]
$$

where the superscript ${ }^{\prime}$ and " denote real and imaginary parts. However, this is not the only underlying field distribution that can give rise to unpolarized light [16]. For instance, by coupling the distribution of the two absolute phases, it is possible to create a distribution that is indistinguishable from that arising from independent Gaussian distributed complex fields without access to the higher order moments of the real and imaginary components of the complex field along the two orthogonal directions [17]. An example of such coupling can be described by a distribution like

$$
p\left(\theta_{x}, \theta_{y}\right)=\frac{2 \pi-\left|\theta_{x}-\theta_{y}\right|}{4 \pi^{2}} \delta\left(\theta_{x}+\theta_{y}\right)
$$

which, under the assumption that the phases and amplitudes are independent, leads to a joint probability distribution of the complex fields of the form

$$
\begin{aligned}
p\left(E_{x}^{\prime}, E_{x}^{\prime \prime}, E_{y}^{\prime}, E_{y}^{\prime \prime}\right)= & \frac{1}{4 \sigma^{4}} \exp \left[-\frac{\left(E_{x}^{\prime}\right)^{2}+\left(E_{x}^{\prime \prime}\right)^{2}+\left(E_{y}^{\prime}\right)^{2}+\left(E_{y}^{\prime \prime}\right)^{2}}{2 \sigma^{2}}\right]+\frac{2 \pi-\left|\tan ^{-1}\left(E_{x}^{\prime \prime} / E_{x}^{\prime}\right)-\tan ^{-1}\left(E_{y}^{\prime \prime} / E_{y}^{\prime}\right)\right|}{2 \pi^{2}} \\
& +\delta\left[\tan ^{-1}\left(E_{x}^{\prime \prime} / E_{x}^{\prime}\right)+\tan ^{-1}\left(E_{y}^{\prime \prime} / E_{y}^{\prime}\right)\right] .
\end{aligned}
$$

It can be shown that both field distributions given in Eqs. (16) and (18) lead to a joint probability distribution of intensities $I_{x, y}=\left|E_{x, y}\right|^{2}$ and phase difference $\Delta=\left|\theta_{x}-\theta_{y}\right|$ of the form

$$
p\left(I_{x}, I_{y}, \Delta\right)=\frac{1}{\alpha^{2}} \exp \left(-\frac{I_{x}+I_{y}}{\alpha}\right) \frac{2 \pi-\Delta}{4 \pi^{2}} ; \quad \Delta \leq 2 \pi ; I_{x} \geq 0 ; I_{y} \geq 0 .
$$

In turn, this produces a uniform distribution of polarization states

$$
p(r, \theta, \phi)=\frac{1}{2 \pi} \delta(r-1) \frac{\sin \phi}{2} ; \quad \theta \in[0,2 \pi] ; \phi \in[0, \pi]
$$

on the surface of the Poincaré sphere, representative to unpolarized radiation. We note that other vector field distributions exist that, on average, have zero normalized Stokes parameters and therefore can be represented as unpolarized radiation [17].

\subsubsection{Point statistics of vector fields}

As already mentioned, the macroscopic model of an unpolarized speckle pattern is actually too simplified in the case of a speckle produced by multiple scattering of coherent light. As pointed out in Refs. [18,19], when a disordered medium is illuminated by monochromatic and perfectly polarized light (laser light), the field at any point in the speckle pattern is 
perfectly polarized, although the state of polarization (elliptic, circular or linear) might be very different from one speckle point to another. Intuitively, two points separated by a sufficiently large distance should have independent polarization states, so that on average over a large area (macroscopic scale) the picture of an unpolarized speckle pattern makes sense. But locally (microscopic scale), the speckle has to be considered as produced by a polarized field.

Statistics of polarized light in speckles produced by multiple scattering have been studied by Cohen et al. [19]. At a given point in the speckle pattern, the elliptically polarized light can be described by the ellipticity $\epsilon$, defined as the ratio between the major and minor axes of the polarization ellipse. The values $\epsilon=0$ and $\epsilon=1$ correspond to linear and circular polarizations, respectively. The statistical distribution $p(\epsilon)$ can be calculated assuming a paraxial observation in the far field, in which the field is a two-dimensional object with two components having complex amplitudes $E_{x}$ and $E_{y}$. In the multiple scattering regime, for an illumination with a linearly polarized field along direction $x$, one can introduce a depolarization parameter $\rho=\left\langle I_{x}\right\rangle /\left\langle I_{y}\right\rangle$, which in the multiple scattering is close to unity [18]. For $\rho=1$, one obtains [19]:

$$
p(\epsilon)=\frac{2\left(1-\epsilon^{2}\right)}{\left(1+\epsilon^{2}\right)^{2}} .
$$

In this distribution, the maximum is obtained for $\epsilon=0$, showing that linear polarization is the most likely polarization state. Another feature is that $p(\epsilon)=0$ for $\epsilon=1$, meaning that circular polarization has vanishing probability. It is also easy to compute the averaged ellipticity $\langle\epsilon\rangle=1-\log 2$.

\subsubsection{Two-point properties of vector fields}

As we discussed previously, optical fields manifest, in general, variations of both coherence and polarization properties. While the Stokes vector elements, defined in terms of second-order field correlations, provide means to characterize the local polarization properties of random fields, higher order correlations are necessary to describe the variability of polarization characteristics. In other words, fourth-order field statistics are needed to quantify the similarity between two different polarization states. Nevertheless, such a two-point quantity can be obtained from a direct generalization of the concept of degree of polarization.

To provide measurable information, the appropriate measure should be independent on the choice of coordinate frame, and it should also reduce to the classical degree of polarization when $\mathbf{r}_{1}=\mathbf{r}_{2}$. To describe the similarity between polarization states at two different locations, one can start from the cross spectral density matrix

$$
\mathbf{W}\left(\mathbf{r}_{1}, \mathbf{r}_{2}\right)=\left(\begin{array}{ll}
\left\langle E_{x}^{*}\left(\mathbf{r}_{1}\right) E_{x}\left(\mathbf{r}_{2}\right)\right\rangle & \left\langle E_{x}^{*}\left(\mathbf{r}_{1}\right) E_{y}\left(\mathbf{r}_{2}\right)\right\rangle \\
\left\langle E_{y}^{*}\left(\mathbf{r}_{1}\right) E_{x}\left(\mathbf{r}_{2}\right)\right\rangle & \left\langle E_{y}^{*}\left(\mathbf{r}_{1}\right) E_{y}\left(\mathbf{r}_{2}\right)\right\rangle
\end{array}\right)
$$

which can be decomposed in the basis formed by the identity matrix and the Pauli spin matrices to obtain

$$
\mathbf{W}\left(\mathbf{r}_{1}, \mathbf{r}_{2}\right)=v_{0}\left(\begin{array}{ll}
1 & 0 \\
0 & 1
\end{array}\right)+v_{1}\left(\begin{array}{cc}
1 & 0 \\
0 & -1
\end{array}\right)+v_{2}\left(\begin{array}{ll}
0 & 1 \\
1 & 0
\end{array}\right)+v_{3}\left(\begin{array}{cc}
0 & -i \\
i & 0
\end{array}\right) .
$$

The parameters

$$
\begin{aligned}
& v_{1}=\frac{J_{11}\left(\mathbf{r}_{1}, \mathbf{r}_{2}\right)-J_{22}\left(\mathbf{r}_{1}, \mathbf{r}_{2}\right)}{2} \\
& v_{2}=\frac{J_{12}\left(\mathbf{r}_{1}, \mathbf{r}_{2}\right)+J_{21}\left(\mathbf{r}_{1}, \mathbf{r}_{2}\right)}{2} \\
& v_{3}=i \frac{J_{12}\left(\mathbf{r}_{1}, \mathbf{r}_{2}\right)-J_{21}\left(\mathbf{r}_{1}, \mathbf{r}_{2}\right)}{2}
\end{aligned}
$$

can be obtained interferometrically via visibility measurements [3]. When $\mathbf{r}_{1}=\mathbf{r}_{2}$, the parameters $v_{i}$ correspond to the conventional Stokes vector elements $(q, u, v)$ defined in Eq. (7). In the traditional theory of polarization, i.e. at a particular point in space, the degree of polarization is defined in terms of measurable intensities as the ratio

$$
P^{2}=4 \frac{q^{2}+u^{2}+v^{2}}{I^{2}}, \quad 0 \leq P \leq 1 .
$$

Similarly, the parameters $v_{i}$ can be used to define a "complex degree of mutual polarization" (CDMP) between the light fluctuations at $\mathbf{r}_{1}$ and $\mathbf{r}_{2}$ as [20]

$$
V^{2}\left(\mathbf{r}_{1}, \mathbf{r}_{2}\right)=4 \frac{v_{1}^{2}+v_{2}^{2}+v_{3}^{2}}{I\left(\mathbf{r}_{1}\right) I\left(\mathbf{r}_{2}\right)} .
$$

We emphasize that the complex degree of mutual polarization $V\left(\mathbf{r}_{1}, \mathbf{r}_{2}\right)$ expresses the relation between polarimetric quantities at two points in terms of measurable visibilities. Furthermore, the CDMP is a complex quantity, containing information in both its phase and magnitude. In the simple case of fully correlated fields, it is easy to see that $|V|$ is a measure of similarity between the states of polarization at two points, the phase is directly related to the phase relationship between the electric field at the two points, and that it is independent on the choice of coordinate frames.

A few cases of correlated fields having spatial variations of the state of polarization are illustrated in Fig. 5 together with the corresponding field correlation matrices and the CDMP values. This situation is typical, for example, in a fully developed 


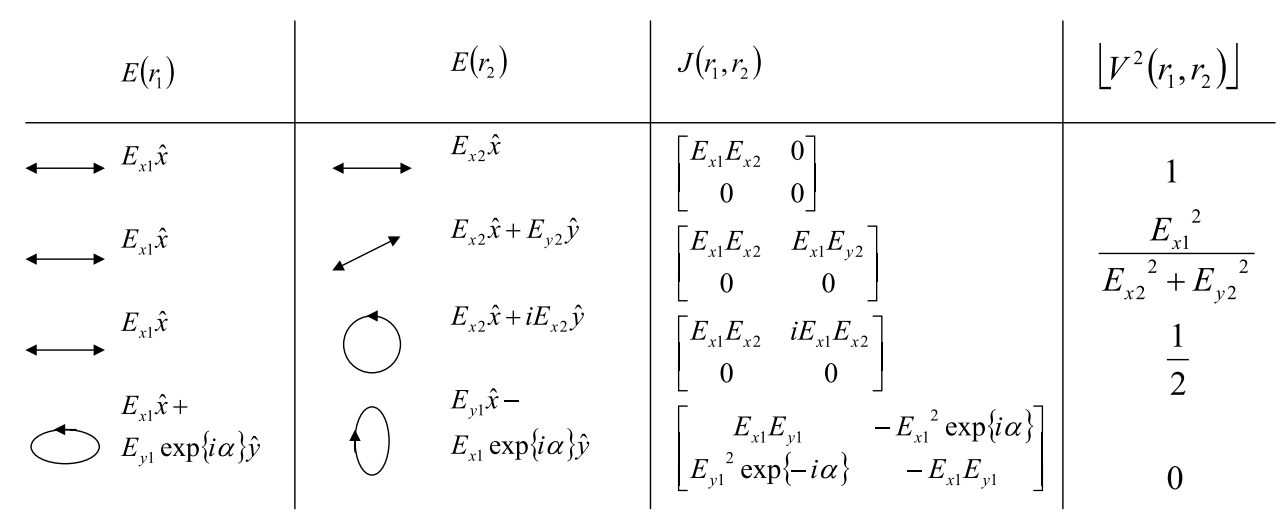

Fig. 5. Field correlation matrix and the complex degree of mutual polarization for different cases of fully correlated fields as indicated in the first two columns.

Source: From Ref. [20].
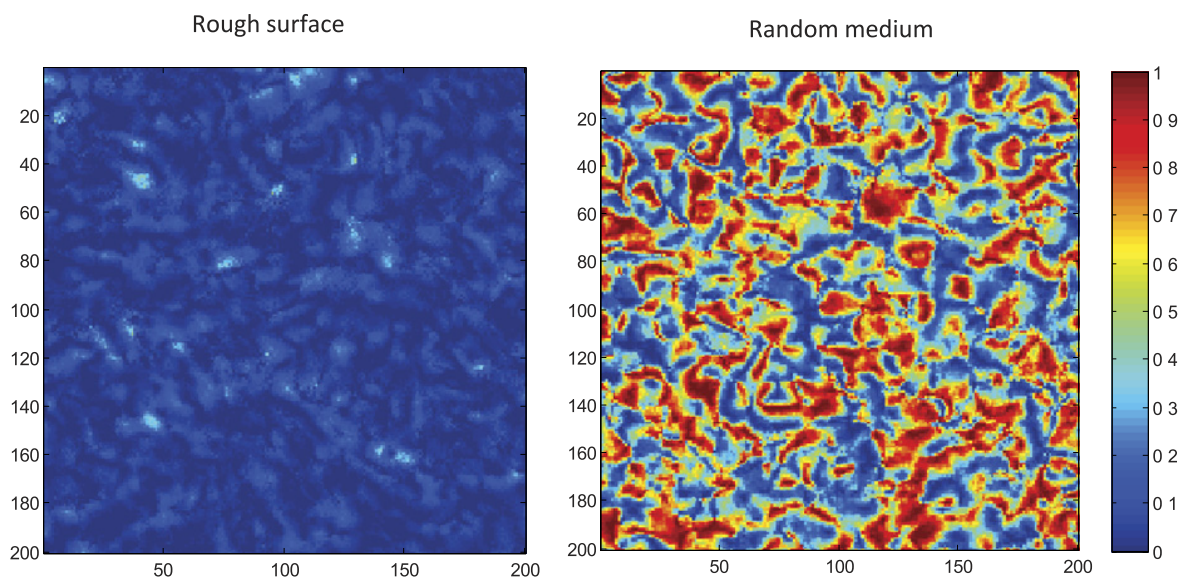

Fig. 6. CDMP maps corresponding to randoms speckle fields originating from scattering from a rough surface and a random medium. The values of $|V|$ are calculated with respect to the incident state of polarization.

speckle pattern where CDMP can be used to measure directly the similarity between the state of polarization at two different points. As can be seen, when the two points are in the same linear state of polarization the magnitude of $V\left(\mathbf{r}_{1}, \mathbf{r}_{2}\right)$ is unity, as one would expect for the same state of polarization. When the two fields have different linear polarizations, the magnitude of $V\left(\mathbf{r}_{1}, \mathbf{r}_{2}\right)$ is a ratio related to how similar the two linear states are, and its phase is the constant phase difference between the fields at $\mathbf{r}_{1}$ and $\mathbf{r}_{2}$. Finally, in the general case of two elliptical states that are orthogonal, $|V|$ is zero.

\subsubsection{Polarimetric analysis of speckle patterns}

Having access to polarimetric information across the entire spatial extent of a random field permits extracting information that is usually overlooked when describing speckle fields obeying a zero-mean Gaussian statistics and only the local intensities are available. Specific features such as the presence of non-stationarities or similarities between different realizations of disorder can be identified using the information contained in CDMP maps. Probability distributions of CDMP values or the extend of their spatial correlations depend on specific characteristics of scattering processes and can be used to trace the origin of the random field. This may be used, for example, to get information about the disordered medium producing the speckle pattern, as illustrated in Fig. 6.

Moreover, CDMP maps such as that shown in Fig. 6 permit quantifying length scales associated with specific physical depolarization process. For instance, in a two dimensional map one could evaluate the decorrelation length of the CDMP by evaluating the decay of $|V(\mathbf{r}, \mathbf{r}+\delta \mathbf{r})|$ around each location $\mathbf{r}$. The original speckle can then be encoded in this CDMP decay length to identify and characterize the extent of different polarization processes, as illustrated in Fig. 7.

As can be seen, having access to polarimetric information across the spatial extent of the field allows building fourth-order joint statistical parameters, which in turn permit identifying quite efficiently field nonstationarities such as the coherent contribution to backscattering [21]. These polarimetric features can be found without any prior knowledge about their extent and, most importantly, they can reveal properties that traditionally are inferred only through ensemble averages [21-23]. 

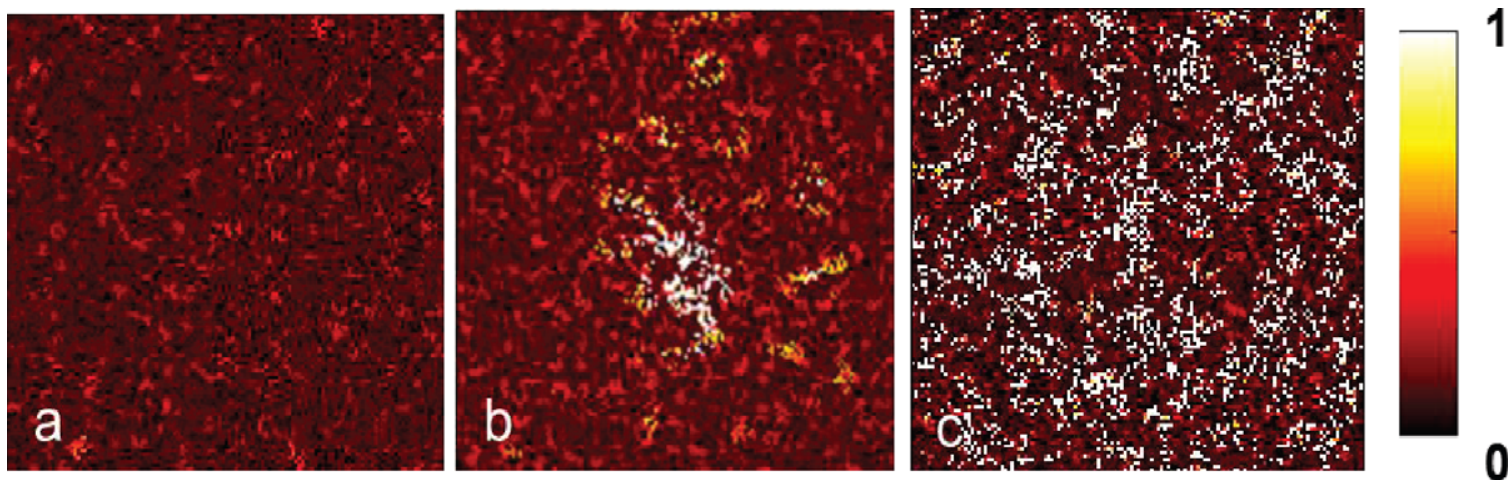

Fig. 7. Spatial distributions of CDMP decay lengths corresponding to backscattering from random media with decreasing values of transport mean free path $\ell^{*}$ (from a to $\mathrm{c}$ ). The distributions of CDMP decay lengths clearly indicate the average extent of the coherent backscattering cone for these media. Note that the CDMP maps are constructed from one single realization of field-matter interaction.

Source: From Ref. [21].

\subsection{Field-field correlations in statistically homogeneous and isotropic media}

In some particular geometries, explicit expressions of field-field correlation functions in speckle patterns can be obtained. The simplest situation corresponds to a speckle pattern produced in the bulk of a disordered medium, that is assumed to be infinite, and statistically homogeneous and isotropic. In this section we compute the cross-spectral density matrix $\mathbf{W}$, and in particular its trace that is often taken as a measure of spatial coherence.

\subsubsection{Model of a disordered medium}

The scattering medium, assumed to be non absorbing, is described by a real dielectric function $\epsilon(\mathbf{r})=\epsilon_{b}+\delta \epsilon(\mathbf{r})$, where $\epsilon_{b}$ is a uniform background and $\delta \epsilon(\mathbf{r})$ is the fluctuating part that satisfies

$$
\begin{aligned}
& \langle\delta \epsilon(\mathbf{r})\rangle=0 \\
& \left\langle\delta \epsilon(\mathbf{r}) \delta \epsilon\left(\mathbf{r}^{\prime}\right)\right\rangle=\frac{A}{\pi^{3 / 2} \ell_{c}^{3}} \exp \left(-\left|\mathbf{r}-\mathbf{r}^{\prime}\right|^{2} / \ell_{c}^{2}\right) .
\end{aligned}
$$

In these expressions the brackets $\langle\ldots\rangle$ denote a statistical average over an ensemble of realizations of the disordered medium. The correlation function of $\delta \epsilon(\mathbf{r})$ is assumed to be Gaussian, and characterized by a correlation length $\ell_{c}$. In a medium made of particles, this correlation length would be at least on the order of the particle size. In the mesoscopic regime, we assume that the following inequalities are satisfied:

$$
\ell_{c} \ll \lambda_{b} \ll \ell_{s}
$$

where $\lambda_{b}$ is the wavelength in the uniform background medium and $\ell_{s}$ is the scattering mean free path. The scattering mean free path is related to the amplitude $A$ of the fluctuations of the dielectric function by $A=6 \pi /\left(k_{0}^{4} \ell_{s}\right)$ with $k_{0}=\omega / c$ the wavevector in vacuum $[24,25]$. Let us also remark that in the limit $\ell_{c} \rightarrow 0$, one would get $\left\langle\delta \epsilon(\mathbf{r}) \delta \epsilon\left(\mathbf{r}^{\prime}\right)\right\rangle=A \delta\left(\mathbf{r}-\mathbf{r}^{\prime}\right)$ which is the so-called white-noise model of disorder that has been used extensively in mesoscopic physics [24].

\subsubsection{Averaged field and scattering mean free path}

An important step before the computation of field-field correlations is the determination of the averaged retarded dyadic Green function $\left\langle\mathbf{G}\left(\mathbf{r}-\mathbf{r}^{\prime}\right)\right\rangle$ in the medium. The averaged Green function obeys the Dyson equation, which can be derived for scalar [24-26] as well as for vector waves [27]. In the particular case of a statistically homogeneous and isotropic medium, with a correlation length satisfying condition (28), the Dyson equation simplifies into a propagation equation in an effective medium described by a local and isotropic dielectric function $\epsilon_{\text {eff }}(\omega)$ [28], that reads

$$
\nabla \times \nabla \times\left\langle\mathbf{G}\left(\mathbf{r}, \mathbf{r}^{\prime}\right)\right\rangle-\epsilon_{\mathrm{eff}}(\omega) k_{0}^{2}\left\langle\mathbf{G}\left(\mathbf{r}, \mathbf{r}^{\prime}\right)\right\rangle=\delta\left(\mathbf{r}-\mathbf{r}^{\prime}\right) .
$$

The computation of the effective dielectric function is a complicated problem. When condition (28) is satisfied, a perturbation theory can be used, leading to explicit and useful results $[25,27,28]$. To lowest order in the perturbative parameter $\left(k_{b} \ell_{s}\right)^{-1}$, the effective dielectric function takes the simple form [29]

$$
\epsilon_{\mathrm{eff}}(\omega)=\epsilon_{b}\left(1+\frac{i}{k_{b} \ell_{s}}\right)
$$

The imaginary part describes the attenuation of the averaged field by scattering (that occurs even in the absence of absorption), with the characteristic decay length $\ell_{s}$. 


\subsubsection{Basic equation for field-field correlation functions}

In the multiple scattering regime, the field correlation function $W_{k l}\left(\mathbf{r}, \mathbf{r}^{\prime}, \omega\right)=\left\langle E_{k}(\mathbf{r}, \omega) E_{l}^{*}\left(\mathbf{r}^{\prime}, \omega\right)\right\rangle$ of two components $k$ and $l$ of the vector electromagnetic field, that constitutes the cross-spectral density matrix $\mathbf{W}$ introduced in Section 1.3, obeys an exact integral equation known as the Bethe-Salpeter equation. Since we focus in this review on the consequences of this equation for the state of coherence and polarization of electromagnetic speckles, we do not reproduce its derivation. The interested reader can find a full derivation in textbooks dedicated to wave scattering or mesoscopic physics [24-28]. Using tensor notations (implicit summation over repeated indices), the Bethe-Salpeter equation reads (the dependence on $\omega$ is omitted for simplicity)

$$
\left\langle E_{k}(\mathbf{r}) E_{l}^{*}\left(\mathbf{r}^{\prime}\right)\right\rangle=\left\langle E_{k}(\mathbf{r})\right\rangle\left\langle E_{l}^{*}\left(\mathbf{r}^{\prime}\right)\right\rangle+\int\left\langle G_{k i}\left(\mathbf{r}, \mathbf{r}_{1}\right)\right\rangle\left\langle G_{l j}^{*}\left(\mathbf{r}^{\prime}, \mathbf{r}_{1}^{\prime}\right)\right\rangle \Gamma_{i j m n}\left(\mathbf{r}_{1}, \mathbf{r}_{1}^{\prime}, \mathbf{r}_{2}, \mathbf{r}_{2}^{\prime}\right)\left\langle E_{m}\left(\mathbf{r}_{2}\right) E_{n}^{*}\left(\mathbf{r}_{2}^{\prime}\right)\right\rangle d^{3} r_{1} d^{3} r_{1}^{\prime} d^{3} r_{2} d^{3} r_{2}^{\prime} .
$$

The kernel $\Gamma_{i j m n}\left(\mathbf{r}_{1}, \mathbf{r}_{1}^{\prime}, \mathbf{r}_{2}, \mathbf{r}_{2}^{\prime}\right)$ is a four-point and four-rank tensor, known as the irreducible vertex (the vocabulary has been borrowed to quantum-field theory). Physically, this vertex describes the building of field-field spatial correlations accounting for all multiple scattering sequences in the disorder medium and their mutual interferences. In practice the irreducible vertex is an object that is difficult to handle and exact analytical computations are out of reach. Approximate perturbation theories (often stated in the form of diagrammatic methods in textbooks) are necessary to get practical results from the Bethe-Salpeter equation.

In the limit $\ell_{s} \gg \lambda_{b}$, a perturbative expansion to lowest order in term of the small parameter $\left(k_{b} \ell_{s}\right)^{-1}$ leads to a simplification of the irreducible vertex that reads [24-26]

$$
\Gamma_{i j m n}\left(\mathbf{r}_{1}, \mathbf{r}_{1}^{\prime}, \mathbf{r}_{2}, \mathbf{r}_{2}^{\prime}\right) \simeq k_{0}^{4}\left\langle\delta \epsilon\left(\mathbf{r}_{1}\right) \delta \epsilon\left(\mathbf{r}_{1}^{\prime}\right)\right\rangle \delta\left(\mathbf{r}_{1}-\mathbf{r}_{2}\right) \delta\left(\mathbf{r}_{1}^{\prime}-\mathbf{r}_{2}^{\prime}\right) \delta_{i m} \delta_{j n} .
$$

This simplification is known as the ladder approximation, since it amounts to keeping only ladder diagrams in the calculation of the field-field correlation function [24-28]. Inserting (32) into (31) leads to

$$
\left\langle E_{k}(\mathbf{r}) E_{l}^{*}\left(\mathbf{r}^{\prime}\right)\right\rangle=\left\langle E_{k}(\mathbf{r})\right\rangle\left\langle E_{l}^{*}\left(\mathbf{r}^{\prime}\right)\right\rangle+k_{0}^{4} \int\left\langle G_{k m}\left(\mathbf{r}, \mathbf{r}_{1}\right)\right\rangle\left\langle G_{l n}^{*}\left(\mathbf{r}^{\prime}, \mathbf{r}_{1}\right)\right\rangle\left\langle\delta \epsilon\left(\mathbf{r}_{1}\right) \delta \epsilon\left(\mathbf{r}_{1}^{\prime}\right)\right\rangle\left\langle E_{m}\left(\mathbf{r}_{1}\right) E_{n}^{*}\left(\mathbf{r}_{1}^{\prime}\right)\right\rangle d^{3} r_{1} d^{3} r_{1}^{\prime} .
$$

This integral equation is the starting point for explicit calculations of field-field correlations in disordered media. It requires the knowledge of the correlation function of the fluctuations of the dielectric function (model of disorder) and of the averaged dyadic Green function (Dyson's equation).

\subsubsection{Degree of spatial coherence in an infinite medium}

The field-field correlation function in Eq. (33) defines the full cross-spectral density matrix $\mathbf{W}$ from which the spatial coherence and polarization properties of the field can be characterized. Spatial coherence can be measured by the trace $\sum_{k}\left\langle E_{k}(\mathbf{r}) E_{k}^{*}\left(\mathbf{r}^{\prime}\right)\right\rangle$ of the matrix $\mathbf{W}$, that averages out the polarization degrees of freedom [30]. The degree of spatial coherence is defined as (using again implicit summation over repeated indices)

$$
\gamma_{E}\left(\mathbf{r}, \mathbf{r}^{\prime}\right)=\frac{\left\langle E_{k}(\mathbf{r}) E_{k}^{*}\left(\mathbf{r}^{\prime}\right)\right\rangle}{\left\langle|\mathbf{E}(\mathbf{r})|^{2}\right\rangle}
$$

where the normalization term $\left\langle|\mathbf{E}(\mathbf{r})|^{2}\right\rangle$ is the averaged intensity in the scattering medium.

In order to compute the field-field correlation function, we insert Eq. (27) into Eq. (33). Moreover, since we are interested in the computation of field-field correlations far from sources, we can neglect the exponentially small contribution of the averaged field (that decays on the scale of $\ell_{s}$ ). This leads to

$$
\left\langle E_{k}(\mathbf{r}) E_{l}^{*}\left(\mathbf{r}^{\prime}\right)\right\rangle=\int_{V}\left\langle G_{k m}\left(\mathbf{r}, \mathbf{r}_{1}\right)\right\rangle\left\langle G_{l n}^{*}\left(\mathbf{r}^{\prime}, \mathbf{r}_{1}^{\prime}\right)\right\rangle C_{m n}\left(\left|\mathbf{r}_{1}-\mathbf{r}_{1}^{\prime}\right|\right) d^{3} r_{1} d^{3} r_{1}^{\prime}
$$

where we have introduced

$$
C_{m n}\left(\left|\mathbf{r}_{1}-\mathbf{r}_{1}^{\prime}\right|\right)=\frac{6 \pi}{\pi^{3 / 2} \ell_{s} \ell_{c}^{3}} \exp \left(-\left|\mathbf{r}_{1}-\mathbf{r}_{1}^{\prime}\right|^{2} / \ell_{c}^{2}\right)\left\langle E_{m}\left(\mathbf{r}_{1}\right) E_{n}^{*}\left(\mathbf{r}_{1}^{\prime}\right)\right\rangle .
$$

Statistical homogeneity and isotropy ensures that $C_{m n}$ depends only on $\left|\mathbf{r}_{1}-\mathbf{r}_{1}^{\prime}\right|$. We now perform the change of variables

$$
\mathbf{R}=\frac{\mathbf{r}_{1}+\mathbf{r}_{1}^{\prime}}{2}, \quad \mathbf{X}=\mathbf{r}_{1}-\mathbf{r}_{1}^{\prime}
$$

with unit Jacobian. This leads to

$$
\left\langle E_{k}(\mathbf{r}) E_{l}^{*}\left(\mathbf{r}^{\prime}\right)\right\rangle=\int\left[\int\left\langle G_{k m}(\mathbf{r}-\mathbf{R}-\mathbf{X} / 2)\right\rangle\left\langle G_{l n}^{*}\left(\mathbf{r}^{\prime}-\mathbf{R}+\mathbf{X} / 2\right)\right\rangle d^{3} R\right] C_{m n}(|\mathbf{X}|) d^{3} X .
$$


In the disordered medium, we assume that the field in unpolarized. This means that $C_{m n}(|\mathbf{X}|)=C_{m m}(|\mathbf{X}|) \delta_{m n}$. The integral in brackets can be simplified using the following identity [31]

$$
k_{0}^{2} \int_{V} \operatorname{Im} \epsilon_{\mathrm{eff}}(\omega)\left\langle G_{k m}\left(\mathbf{r}, \mathbf{r}^{\prime \prime}\right)\right\rangle\left\langle G_{l m}^{*}\left(\mathbf{r}^{\prime}, \mathbf{r}^{\prime \prime}\right)\right\rangle d^{3} r^{\prime \prime}=\operatorname{Im}\left\langle G_{k l}\left(\mathbf{r}, \mathbf{r}^{\prime}\right)\right\rangle
$$

and we end up with

$$
\left\langle E_{k}(\mathbf{r}) E_{l}^{*}\left(\mathbf{r}^{\prime}\right)\right\rangle=\frac{1}{k_{0}^{2} \operatorname{Im} \epsilon_{\text {eff }}(\omega)} \int \operatorname{Im}\left\langle G_{k l}\left(\mathbf{r}-\mathbf{r}^{\prime}-\mathbf{X}\right)\right\rangle C_{m m}(|\mathbf{X}|) d^{3} X .
$$

From Eqs. (30), (34) and (40) we get

$$
\gamma_{E}\left(\mathbf{r}, \mathbf{r}^{\prime}\right)=\frac{1}{\left\langle|\mathbf{E}(\mathbf{r})|^{2}\right\rangle} \frac{\ell_{s}}{k_{b}} \int \operatorname{Im}\left\langle G_{k k}\left(\mathbf{r}-\mathbf{r}^{\prime}-\mathbf{X}\right)\right\rangle C_{m m}(|\mathbf{X}|) d^{3} X
$$

The remaining integral takes the form of a convolution product. The averaged dyadic Green function is the solution of Eq. (29) in a homogeneous medium with dielectric function $\epsilon_{\mathrm{eff}}(\omega)$. Its expression is given by

$$
\left\langle\mathbf{G}\left(\mathbf{r}-\mathbf{r}^{\prime}\right)\right\rangle=\left[\mathbf{I}+\frac{1}{k_{\text {eff }}^{2}} \nabla \nabla\right] \frac{\exp \left(i k_{\mathrm{eff}} \rho\right)}{4 \pi \rho} \text { for } \rho \neq 0
$$

where $\rho=\left|\mathbf{r}-\mathbf{r}^{\prime}\right|$ and $k_{\text {eff }}=\epsilon_{\text {eff }}(\omega) k_{0}$. The trace of the averaged Green function varies on scales of $\lambda_{b}$ and $\ell_{s}[32,33]$, while the correlation function $C_{m n}(|\mathbf{X}|)$ varies on the scale of $\ell_{c}$. Since $\ell_{c} \ll \lambda_{b} \ll \ell_{s}$, the convolution product can be simplified:

$$
\gamma_{E}\left(\mathbf{r}, \mathbf{r}^{\prime}\right)=\frac{1}{\left\langle|\mathbf{E}(\mathbf{r})|^{2}\right\rangle} \frac{6 \pi}{k_{b}} \operatorname{Im}\left\langle G_{k k}\left(\mathbf{r}-\mathbf{r}^{\prime}\right)\right\rangle \int \frac{\exp \left(-|\mathbf{X}|^{2} / \ell_{c}^{2}\right)}{\pi^{3 / 2} \ell_{c}^{3}}\left\langle E_{m}(0) E_{m}^{*}(\mathbf{X})\right\rangle d^{3} X .
$$

The Gaussian function acts as a Dirac delta function with respect to the field-field correlation function. We end up with

$$
\gamma_{E}\left(\mathbf{r}, \mathbf{r}^{\prime}\right)=\frac{2 \pi}{k_{b}} \operatorname{Im}\left\langle G_{k k}\left(\mathbf{r}-\mathbf{r}^{\prime}\right)\right\rangle .
$$

From Eq. (42), the imaginary part of the averaged dyadic Green function can be computed explicitly, and we finally get

$$
\gamma_{E}(\rho)=\operatorname{sinc}\left(\frac{2 \pi \rho}{\lambda_{b}}\right) \exp \left(-\frac{\rho}{2 \ell_{S}}\right) .
$$

We conclude by recalling that this expression is valid in any disordered medium that is statistically homogeneous and isotropic, in the limit $\ell_{c} \ll \lambda_{b} \ll \ell_{s}$. It relies on the assumption of a field varying on a scale larger than $\ell_{c}$, a condition of validity of the effective medium model that we have used in the first place in the Dyson equation (29).

\subsubsection{Analogy with blackbody radiation}

The form of the degree of spatial coherence given by Eq. (45) is the same as that obtained for scalar waves [25,34]. In practice, since the derivation is valid in the regime $\ell_{s} \gg \lambda_{b}$, the influence of the exponential term is negligible and the degree of spatial coherence is chiefly driven by the function $\operatorname{sinc}\left(2 \pi \rho / \lambda_{b}\right)$, leading to a spatial coherence length $\lambda_{b} / 2$. This result is independent on the internal structure of the disordered medium, thus being universal, in the same way as the Rayleigh statistics for the intensity of a fully developed speckle is independent on the system that produces this speckle.

This expression of the degree of spatial coherence is also found in the case of equilibrium thermal radiation (blackbody radiation) in a homogeneous medium [35]. A relation similar to Eq. (44) is derived in this case, as a direct consequence of the fluctuation-dissipation theorem [36]. The use of fluctuation-dissipation relations leads to a general method for calculating field-field correlation functions at equilibrium in more complex geometries, such as cavities or close to interfaces, by using the appropriate Green function $[37,38]$. This suggests that in the case of speckle correlations, the approach presented here could also be extended to more complex geometries. The semi-infinite geometry can be handled in analogy to the thermal fluctuation approach, as shown in Ref. [39]. This is the subject of the next section.

The degree of spatial coherence given by Eq. (45) actually reflects a universal behavior of field-field correlations, when the field fluctuations are statistically homogeneous and isotropic, as pointed out in Ref. [40]. Speckle fluctuations produced by multiple scattering in an infinite medium, or thermal fluctuations in a homogeneous medium, are two situations that belong to this class of statistical processes and therefore exhibit those universal properties.

\subsubsection{Spatial coherence and cross density of states}

We have seen that a connection between the field-field spatial correlation function and the imaginary part of a Green function is observed in many different situations where the spatial coherence of random fields (produced by random sources 
and/or a disordered medium) needs to be characterized, as in speckle patterns [25] or thermal radiation fields [38]. The same formal relationship between field-field correlation functions and Green functions is found for seismic and acoustic waves, and is at the origin of the Green function retrieval technique based on noise correlations [41]. Indeed, the imaginary part of the dyadic Green function at two different points characterizes the intrinsic spatial coherence of the medium at a given frequency $\omega$, sustained by the underlying structure of eigenmodes, as shown recently by Cazé et al. [42]. One can introduce a two-point quantity $\rho\left(\mathbf{r}, \mathbf{r}^{\prime}, \omega\right)$, referred to as Cross Density of States (CDOS), and defined as

$$
\rho\left(\mathbf{r}, \mathbf{r}^{\prime}, \omega\right)=\frac{2 \omega}{\pi c^{2}} \operatorname{Im}\left[\operatorname{Tr} \mathbf{G}\left(\mathbf{r}, \mathbf{r}^{\prime}, \omega\right)\right] .
$$

In this expression, $c$ is the speed of light in vacuum, and $\mathbf{G}\left(\mathbf{r}, \mathbf{r}^{\prime}, \omega\right)$ is the electric dyadic Green function of the medium. The prefactor in the definition of the CDOS has been chosen so that it reduces to the electric part of the Local Density Of States (LDOS) when $\mathbf{r}$ and $\mathbf{r}^{\prime}$ coincide [43]. The CDOS can be understood as a counting of the electromagnetic eigenmodes that connect two different points at a given frequency, the counting being weighted by the strength of each eigenmode $[42,44]$. In a network picture, the CDOS measures the number of channels connecting two points. The LDOS measures the number of channels crossing at a given point. For equilibrium thermal radiation, field-field spatial correlation functions are driven by the CDOS, while the energy density at one point is given by the LDOS [38]. The result given in Eq. (45) also puts forward the role of the CDOS. Here the field-field spatial correlation function is given by the averaged CDOS (the averaging being over the medium itself, it applies to the Green function and the CDOS, which is not the case for thermal radiation for which the averaging process is over the fluctuations of the thermal sources). Finally let us remark that the CDOS also enters the description of the process of focusing by time reversal in a closed cavity, that is also intimately connected to field-field correlations [45,46].

\subsubsection{Full cross-spectral density matrix}

On average over an ensemble of realizations of a disordered medium, multiple scattering is expected to depolarize a perfectly polarized incident wave. In the optical regime, depolarization of scattered light has been observed in reflection either in the study of coherent backscattering [47,48] or in dynamic speckles [49-52]. On the theoretical aspects, depolarization can be studied using perturbation theory [in the small parameter $\left(k_{0} \ell_{s}\right)^{-1}$ ] based on the vector wave equation [53-57], transfer matrix approaches [58,59] or vector radiative transfer or random walk calculations [60-62]. A perturbative calculation of the full cross-spectral density matrix $\mathbf{W}$ can be performed in the bulk of an uncorrelated disordered medium, under illumination by a monochromatic electric-dipole point source [63]. The calculation is based on the Bethe-Salpeter equation (33), but all elements $W_{j k}\left(\mathbf{r}, \mathbf{r}^{\prime}, \omega\right)=\left\langle E_{j}(\mathbf{r}, \omega) E_{k}^{*}\left(\mathbf{r}^{\prime}, \omega\right)\right\rangle$ are calculated, and not only the trace as in Section 2.3.4. The calculation is technically involved since one needs to solve the Bethe-Salpeter equation. This can be done for a scattering medium with uncorrelated disorder. Such a medium is described by a white-noise model, using a dielectric function of the form $\epsilon(\mathbf{r})=1+\delta \epsilon(\mathbf{r})$ with

$$
\begin{aligned}
& \langle\delta \epsilon(\mathbf{r})\rangle=0 \\
& \left\langle\delta \epsilon(\mathbf{r}) \delta \epsilon\left(\mathbf{r}^{\prime}\right)\right\rangle=\frac{6 \pi}{k_{0}^{4} \ell_{s}} \delta\left(\mathbf{r}-\mathbf{r}^{\prime}\right) .
\end{aligned}
$$

Within this model a perturbative calculation leads to analytical results that we summarize here. The elements of the crossspectral density matrix can be written in the form

$$
W_{j k}(\mathbf{R}, \mathbf{X})=\left\langle E_{j}(\mathbf{R}+\mathbf{X} / 2) E_{k}^{*}(\mathbf{R}-\mathbf{X} / 2)\right\rangle
$$

where $\mathbf{R}$ is the position vector defining the center of the observation region, the origin of coordinates coinciding with the illuminating point source, and $\mathbf{R} \pm \mathbf{X} / 2$ define the two observation points around position $\mathbf{R}$. Without loss of generality, one can take direction $j=k=1$ to coincide with the direction of vector $\mathbf{X}$, and the result reads [63]

$$
W_{j k}(\mathbf{R}, \mathbf{X})= \begin{cases}\frac{f^{(0)}(\mathbf{X})}{8 \pi^{2} \ell_{s} R} & (j=k=1) \\ \frac{f^{(1)}(\mathbf{X})-f^{(0)}(\mathbf{X})}{16 \pi^{2} \ell_{s} R} & (j=k=2,3) \\ 0 & (j \neq k),\end{cases}
$$

with

$$
f^{(0)}(\mathbf{X})=\frac{1}{2\left(k_{0} X\right)^{3}\left(k_{0} \ell\right)}\left[2-k_{0} \ell n_{\mathrm{eff}}^{2}\left(k_{0} X n_{\mathrm{eff}}^{*}-i\right) \exp \left[-i k_{0} X n_{\mathrm{eff}}^{*}\right]-k_{0} \ell n_{\mathrm{eff}}^{\star 2}\left(k_{0} X n_{\mathrm{eff}}+i\right) \exp \left[i k_{0} X n_{\mathrm{eff}}\right]\right]
$$

and

$$
f^{(1)}(\mathbf{X})=\frac{1}{2 i k_{0} X}\left(\exp \left[i k_{0} X n_{\mathrm{eff}}\right]-\exp \left[-i k_{0} X n_{\mathrm{eff}}^{*}\right)\right),
$$

where $n_{\text {eff }}=1+i /\left(2 k_{0} \ell_{s}\right)$ is the effective refractive index of the scattering medium. These expressions are valid to first order in the small parameter $\left(k_{0} \ell_{s}\right)^{-1}$. 
Since the cross-spectral density matrix $\mathbf{W}$ is the building block for the analysis of coherence and polarization in the speckle field, one can study from Eq. (49) the degree of coherence and polarization, for example, at a given distance $R=|\mathbf{R}|$ from a linearly polarized point source. Taking the normalized trace of $\mathbf{W}$ as a measure of the degree of spatial coherence, one recovers the same result in Eq. (45). Regarding the evolution of the degree of polarization, one can study the evolution of the energy density $W_{j k}(\mathbf{R}, \mathbf{X}=0)$ versus the distance $R$ to the point source. One finds that the energy density associated to different states of polarizations is attenuated exponentially with a decay length which, for an uncorrelated medium, is on the order of $\ell_{s}$. In the presence of correlations in the disorder, one could expect the characteristic decay length to be replaced by the transport mean free path $\ell^{*}$, but a derivation remains to be done. The only contribution that is not attenuated corresponds to the unpolarized mode. This is the contribution that survives at large distances, supporting the picture that on average over the configurations of the medium multiple scattering induces a depolarization of the incident light. Let us recall that under coherent illumination, the speckle generated on a single realization of disorder is perfectly polarized, although the state of polarization changes from on speckle spot to the other, so that the depolarization effects discussed here only makes sense after ensemble or spatial averaging.

\subsubsection{Transport models for spatial coherence and polarization}

We have shown how practical calculations of the cross-spectral density matrix and of the degree of spatial coherence can be handled in the multiple scattering regime. Starting from the Bethe-Salpeter equation, explicit expressions can be obtained in the limit $k_{0} \ell_{s} \gg 1$ for statistically homogeneous media with uncorrelated (or weakly correlated) disorder. In this limit, it is known that a transport equation for the specific intensity, known as the radiative transfer equation (RTE), can be obtained as an approximation of the Bethe-Salpeter equation (Eq. (31)). Although historically the RTE was derived as a transport equation for the intensity in the context of energy transfer [64], its derivation in multiple scattering theory leads to a much broader interpretation. Indeed, the specific intensity in this context is defined as the Wigner transform of the field, or in other words as the Fourier transform of the spatial field-field correlation function. Writing the Bethe-Salpeter equation in terms of the specific intensity, and using the ladder approximation (Eq. (33)), leads to the RTE [26]. In this derivation, the resulting RTE is naturally understood as a transport equation for the spatial field-field correlation. An extensive discussion of this wave aspect of the RTE is given in Ref. [65]. The RTE has been used to study the transport of spatial coherence of a beam incident on a multiple scattering medium in the scalar approximation [66]. The vector form of the RTE has been used to study depolarization by multiple scattering in case of Rayleigh scatterers [61], or under the assumption of strong forward scattering [62]. Monte-Carlo simulations with polarized light have also been used to support experimental data [52], and such simulations actually amount to solving the RTE numerically. The use of a radiative transfer equation for the full cross-spectral density matrix, that would allow a description of 3D coherence and polarization, is still a subject of current research. In the simplified diffusion-approximation model, that amounts to performing a large scale asymptotics of the RTE, a transport equation can be found [63], that leads to the results summarized in Section 2.3.7.

\section{Beyond universality in electromagnetic speckle patterns}

\subsection{Degree of spatial coherence above an interface}

In many situations of practical interest, the speckle properties are measured outside the scattering medium, either in the far field or close to the output surface. The properties of coherence and polarization of radiation including the evanescent waves in the proximity of an interface have been discussed in Refs. [67,68]. In this section, we show how the method introduced in Section 2.3 can be extended to geometries involving interfaces, following the approach introduced in Refs. $[39,69]$. The simplest situation is that of a flat interface separating a semi-infinite scattering medium from a vacuum. The interface defines the plane $z=0$, as shown in the inset in Fig. 8 .

\subsubsection{General expression for semi-infinite geometry}

Our starting point is the Bethe-Salpeter equation in the ladder approximation Eq. (33), in which we will remove the exponentially small contribution of the averaged field (the semi-infinite geometry models a thick scattering medium illuminated in transmission, with thickness $L \gg \ell_{S}$, so that the contribution of the averaged field $\langle\mathbf{E}\rangle \sim \exp \left(-L / \ell_{S}\right)$ is negligible). In this equation, the averaged dyadic Green function now corresponds to the solution of the Dyson equation (29) in the appropriate geometry, that corresponds to a semi-infinite medium with homogeneous, local and isotropic dielectric function $\epsilon_{\text {eff }}(\omega)$ filling the half-space $z<0$, while the half-space $z>0$ is a vacuum. The dyadic Green function in that geometry is known [70]. For a source point $\mathbf{r}^{\prime}$ inside the medium and an observation point $\mathbf{r}$ in vacuum, it reads [71]

$$
\left\langle\mathbf{G}\left(\mathbf{r}, \mathbf{r}^{\prime}\right)\right\rangle=\frac{i}{8 \pi^{2}} \int \frac{1}{\gamma_{1}}\left(\mathbf{s} t_{s} \mathbf{s}+\mathbf{p}_{0} t_{p} \mathbf{p}_{1}\right) \exp \left[i \mathbf{K} \cdot\left(\mathbf{R}-\mathbf{R}^{\prime}\right)\right] \exp \left(i \gamma_{0} z-i \gamma_{1} z^{\prime}\right) \mathrm{d}^{2} K
$$

where $\mathbf{R}=(x, y)$ and $\mathbf{K}$ is the component of the wavevector along the interface. The dyadic terms describe the polarization behavior at the interface, with $\mathbf{s}=\hat{\mathbf{K}} \times \hat{z}, \mathbf{p}_{j}=\left(K \hat{z}+\gamma_{j} \hat{\mathbf{K}}\right) / k_{j}$, with $j=0$ (vacuum) or $j=1$ (medium), the symbol ${ }^{\wedge}$ denoting a unit vector and $K=|\mathbf{K}|$. The components of the wavevector along the $z$ direction are $\gamma_{j}=\left(k_{j}^{2}-K^{2}\right)^{1 / 2}$, with $k_{1}=\sqrt{\epsilon_{\text {eff }}(\omega)} k_{0}$, and the determination $\operatorname{Re}\left(\gamma_{j}\right)>0$ and $\operatorname{Im}\left(\gamma_{j}\right)>0 . t_{s}(K)$ and $t_{p}(K)$ are the Fresnel transmission factors 


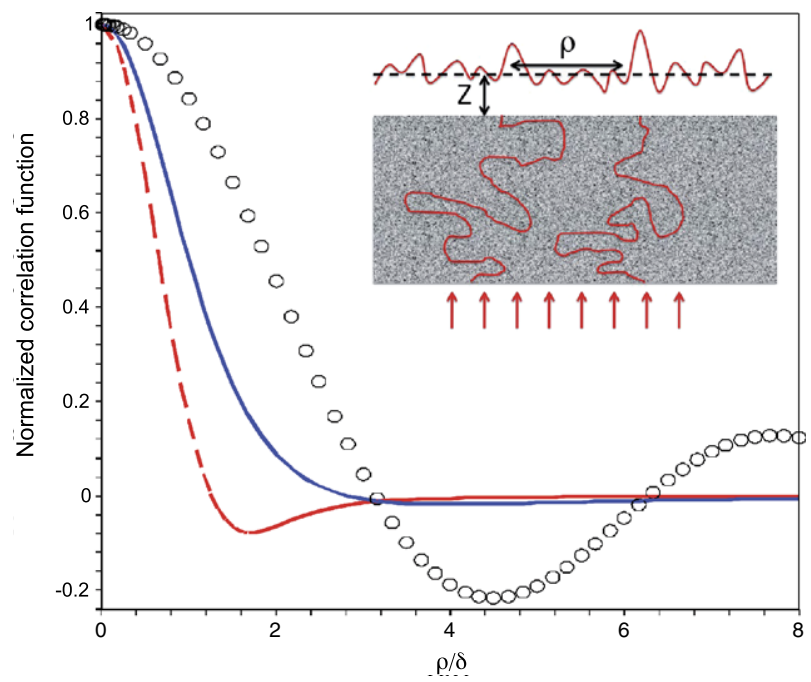

Fig. 8. Degree of spatial coherence $\gamma_{E}\left(\mathbf{r}, \mathbf{r}^{\prime}\right)$ in a plane at a distance $z$ versus $\rho / \delta$, where $\rho$ is the distance between two observation points in a plane at a constant distance $z$. $\delta$ is a reference length scale that is not the same in the far-field and near-field regimes. Black markers: Far-field regime $(z \gg \lambda)$, $\delta=\lambda / 2 \pi$. Blue solid line: near-field regime $\left(\ell_{c} \ll z \ll \lambda\right), \delta=z$. Red dashed line: extreme near-field regime $\left(z \simeq \ell_{c} \ll \lambda\right), \delta=\ell_{c}$.

Source: From Ref. [39].

for $s$ and p-polarized waves [72]. For $z>0$ (upper medium) the plane-wave expansion (52) possesses both propagating waves and evanescent waves. The latter correspond to high spatial frequencies $K>k_{0}$ and imaginary values of $\gamma_{0}$. These evanescent waves dominate in the near field, at distances $z \ll \lambda$ from the interface. The role of these evanescent fields is at the core of near-field optics [73,74]. As we shall see, they also influence electromagnetic near field speckles, leading to statistical properties that may strongly differ from that of far-field speckles. Accounting for evanescent near-field components is a major difference between the approach presented here, and that in Ref. [75] that also addressed the calculation of speckle correlations above an interface using a scalar diffusion approximation. The latter does not account for near-field scattering and cannot lead to reliable results at distances from the surface smaller than the wavelength.

The integral term in Eq. (33) involves the field-field spatial correlation matrix in the scattering medium. We will assume that it remains the same as that in the infinite medium, such that $\left\langle E_{m}\left(\mathbf{r}_{1}\right) E_{n}^{*}\left(\mathbf{r}_{1}^{\prime}\right)\right\rangle=\left\langle E_{m}\left(\mathbf{r}_{1}\right) E_{m}^{*}\left(\mathbf{r}_{1}^{\prime}\right)\right\rangle \delta_{m n}$, with $\left\langle E_{m}\left(\mathbf{r}_{1}\right) E_{m}^{*}\left(\mathbf{r}_{1}^{\prime}\right)\right\rangle$ varying on length scales $\lambda_{b}$ and $\ell_{s}$ [see Eq. (44)]. Since these length scales are much larger than $\ell_{c}$, one can make the approximation $\left\langle\delta \epsilon\left(\mathbf{r}_{1}\right) \delta \epsilon\left(\mathbf{r}_{1}^{\prime}\right)\right\rangle\left\langle E_{m}\left(\mathbf{r}_{1}\right) E_{m}^{*}\left(\mathbf{r}_{1}^{\prime}\right)\right\rangle \simeq\left\langle\delta \epsilon\left(\mathbf{r}_{1}\right) \delta \epsilon\left(\mathbf{r}_{1}^{\prime}\right)\right\rangle\left\langle|\mathbf{E}|^{2}\right\rangle / 3$ in Eq. (33). Under this approximation, using Eq. (52) into Eq. (33) leads to a general expression of the trace of the field-field correlation tensor (that after normalization gives the degree of spatial coherence):

$$
\left\langle E_{k}(\mathbf{r}) E_{k}^{*}\left(\mathbf{r}^{\prime}\right)\right\rangle=\frac{\left\langle|\mathbf{E}|^{2}\right\rangle}{4 \ell_{s}} \int_{0}^{\infty} f(K, z) \exp \left[-\left(K^{2}+\left(\operatorname{Re} \gamma_{1}\right)^{2}\right) \ell_{c}^{2} / 4\right] J_{0}(K \rho) d K
$$

where $J_{0}$ is the ordianry Bessel function and $\rho=\left|\mathbf{R}-\mathbf{R}^{\prime}\right|$ and $K=|\mathbf{K}|$. Note that $\left\langle|\mathbf{E}|^{2}\right\rangle$ in this equation is the averaged intensity in the infinite medium. The function in the integrand is

$$
f(K, z)=\frac{K}{2 \operatorname{Im} \gamma_{1}}\left[\frac{\left|t_{s}\right|^{2}}{\left|\gamma_{1}\right|^{2}}+\frac{\left(K^{2}+\left|\gamma_{0}\right|^{2}\right)\left(K^{2}+\left|\gamma_{1}\right|^{2}\right)}{\left|\sqrt{\epsilon_{\text {eff }}(\omega)}\right|^{2} k_{0}^{4}} \frac{\left|t_{p}\right|^{2}}{\left|\gamma_{1}\right|^{2}}\right] \exp \left(-2 \operatorname{Im} \gamma_{0} z\right)
$$

Eq. (53) is the starting point for explicit calculations of the degree of spatial coherence in a plane at any distance $z$ from the interface, including the near-field region. The function $f(K, z)$ describes both wave transmission at the interface between the effective medium and the observation plane (with a symmetry breaking that influences the polarization state since $s$ and $p$ components behave differently), and the attenuation of the high spatial frequency components (evanescent waves) when $z$ increases, through the term $\exp \left(-2 \operatorname{Im} \gamma_{0} z\right)$ that behaves as a low-pass filter with respect to $K$.

In this model, the use of a local effective dielectric constant $\epsilon_{\text {eff }}(\omega)$ might be questioned, since this requires to disregard field variations on scales that are not larger than $\ell_{c}$ (see Section 2.3.2). The term $\exp \left(-K^{2} \ell_{c}^{2} / 4\right)$ in the integral in Eq. (53) prevents spatial frequencies $K>1 / \ell_{c}$ to play a role, so that rapid variations of the field are automatically filtered out. Therefore, the model remains reliable for distances $z \geq \ell_{c}$. Below this length scale, the non-locality in the effective dielectric function entering the Dyson equation cannot be avoided. The use of non-local models for the effective dielectric function of scattering media is by itself a topic of current study [76].

\subsubsection{Far-field regime}

The general expression (53) can be computed numerically. It is also possible to derive explicit asymptotic expressions that are valid in regimes of relevance for experiments. We first address the far-field regime. It is important to note that by far 
field we mean a measurement in real space in a plane at a given distance $z \gg \lambda$ from the interface ( $\lambda$ being the wavelength in vacuum), for which only propagating components in the plane-wave expansion of the averaged Green function (52) contribute. In practice, a distance $z \sim \lambda$ is already enough to loose the evanescent components, and to give rise to a far-field speckle (here by "near field" we mean the region in which evanescent waves contribute substantially) [73].

In the regime $z \gg \lambda \gg \ell_{c}$, the term $\exp \left(-2 \operatorname{Im} \gamma_{0} z\right)$ reduces the range of $f(K, z)$ to $0 \leq K \leq k_{0}$ (showing that only propagating waves contribute), and in this range the exponential term $\exp \left[-\left(K^{2}+\left(\operatorname{Re} \gamma_{1}\right)^{2}\right) \ell_{c}^{2} / 4\right] \simeq 1$ has a negligible influence in Eq. (53). One obtains:

$$
\left\langle E_{k}(\mathbf{r}) E_{k}^{*}\left(\mathbf{r}^{\prime}\right)\right\rangle=\frac{\left\langle|\mathbf{E}|^{2}\right\rangle}{2 k_{0}} \int_{0}^{k_{0}} \frac{K}{\sqrt{k_{0}^{2}-K^{2}}}[1-R(K)] J_{0}(K \rho) d K
$$

where $R(K)=\left[\left|r_{s}(K)\right|^{2}+\left|r_{p}(K)\right|^{2}\right] / 2$ is the intensity Fresnel reflection factor averaged over polarizations. Neglecting the dependence of $R$ on $K$ (which is rigorously valid only if $\epsilon_{b} \simeq 1, \epsilon_{b}$ being the dielectric function of the background medium constituting the scattering material), the integral simplifies to give

$$
\left\langle E_{k}(\mathbf{r}) E_{k}^{*}\left(\mathbf{r}^{\prime}\right)\right\rangle=\frac{(1-R)\left\langle|\mathbf{E}|^{2}\right\rangle}{2} \operatorname{sinc}\left(k_{0} \rho\right)
$$

which after normalization gives the far-field expression of the degree of spatial coherence

$$
\gamma_{E}\left(\mathbf{r}, \mathbf{r}^{\prime}\right)=\operatorname{sinc}\left(k_{0} \rho\right) \text { for } z \gg \lambda
$$

with $k_{0}=2 \pi / \lambda, \rho=\left|\mathbf{R}-\mathbf{R}^{\prime}\right|$ being the distance between two observation points in a plane at a given distance $z \gg \lambda$ from the interface. The far-field degree of spatial coherence is plotted in Fig. 8 (black markers), versus the normalized distance $2 \pi \rho / \lambda$.

The $\operatorname{sinc}\left(k_{0} \rho\right)$ behavior is identical to that obtained for thermal equilibrium (blackbody) radiation in the far field of a planar thermal source $[77,78]$. It gives a coherence length $\lambda / 2$, that also measures the averaged size of a speckle spot in the far field. The $\operatorname{sinc}\left(k_{0} \rho\right)$ dependence of the far field degree of spatial coherence computed in vacuum is also similar to that obtained inside an infinite medium (Eq. (45)), except that the $\exp \left(-\rho / 2 \ell_{s}\right)$ term is absent. This exponential decay is irrelevant for a computation outside the scattering medium. The far-field degree of spatial coherence also exhibits a universal behavior, since it depends only on the illumination wavelength $\lambda$.

\subsubsection{Near-field regime}

When $z \ll \lambda$, one enters the near-field regime for which the contribution of evanescent waves, corresponding to high spatial frequencies $K$, dominates. The integrand in Eq. (53) can be expanded to leading order in the limit $K \gg k_{0}$, known as the quasi-static limit [73]. This leads to

$$
\left\langle E_{k}(\mathbf{r}) E_{k}^{*}\left(\mathbf{r}^{\prime}\right)\right\rangle=\frac{2\left\langle|\mathbf{E}|^{2}\right\rangle}{k_{0}^{4} \ell_{s}\left|\epsilon_{\mathrm{eff}}(\omega)+1\right|^{2}} \int_{0}^{\infty} K^{2} \exp (-2 K z) \exp \left(-K^{2} \ell_{c}^{2} / 4\right) J_{0}(K \rho) d K
$$

Let us first consider the regime $\ell_{c} \ll z \ll \lambda$, and neglect the influence of the exponential term $\exp \left(-K^{2} \ell_{c}^{2} / 4\right)$, compared to that of $\exp (-2 \mathrm{Kz})$ that acts as a filter. Physically, this means that the observation distance $z$ remains too large for the speckle to be influenced by the internal structure of the disordered medium. In this case, the integral in (58) can be calculated analytically [79]. We end up with

$$
\gamma_{E}\left(\mathbf{r}, \mathbf{r}^{\prime}\right)=\frac{1-\rho^{2} /\left(8 z^{2}\right)}{\left[1+\rho^{2} /\left(4 z^{2}\right)\right]^{5 / 2}} \quad \text { for } \ell_{c} \ll z \ll \lambda .
$$

The near-field degree of spatial coherence is plotted in Fig. 8 (blue solid line), for a fixed distance $z$. Its width, that determines the spatial coherence length, is on the order of the observation distance $z$. This result is a feature of the quasi-static regime, in which the spatial structure of the field is that of an electrostatic field, and therefore is driven by geometrical length scales (and no more by the wavelength) [73]. As another consequence of the analogy between speckle correlations produced by multiple scattering and correlations in thermal radiation fields, the same behavior has been obtained for near fields generated by thermal sources $[78,79]$.

\subsubsection{Extreme near field}

The decrease of the spatial coherence with the observation distance $z$ predicted by Eq. (59) should saturate when $z \simeq \ell_{c}$, i.e., when the distance is such that the internal structure of the disordered material is expected to directly influence the structured of the field. Although the approach developed here is valid only when field variations remain in principle much larger than $\ell_{c}$, it is instructive to push the model to the limit. When $z \simeq \ell_{c}$, the influence of the exponential cut-off $\exp \left(-K^{2} \ell_{c}^{2} / 4\right)$ in Eq. (58) dominates, and a transition is expected to a regime in which the degree of spatial coherence becomes independent on $z$. Neglecting the influence of the term $\exp (-2 K z)$ in the integral, Eq. (58) gives 

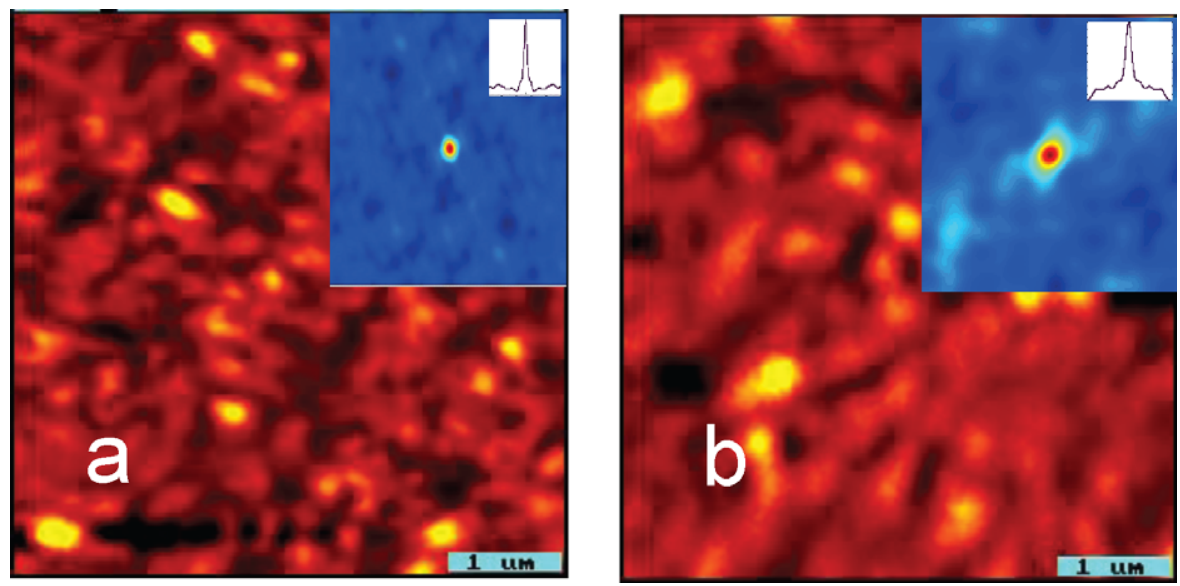

Fig. 9. Typical near- (a) and far-field (b) speckle distributions recorded on scattering from a porous material (Vycor porous glass) with a $28 \%$ void volume and a $4 \mathrm{~nm}$ average pore size. The speckles are recorded by SNOM at a distance $z=20 \mathrm{~nm}$ and $z=5000 \mathrm{~nm}$ from the surface, respectively. The sample is illuminated in transmission at a wavelength $\lambda=488 \mathrm{~nm}$. The detection is performed with an aperture probe, made of an Al-coated fused-silica fiber tip. Also shown are the corresponding autocorrelation images.

$$
\gamma_{E}\left(\mathbf{r}, \mathbf{r}^{\prime}\right)=M\left(\frac{3}{2}, 1, \frac{-\rho^{2}}{\ell_{c}^{2}}\right) \quad \text { for } z \simeq \ell_{c} \ll \lambda
$$

where $M(a, b, x)$ is the confluent hypergeometric function. Let us point out again that due to the analogy with thermal radiation, this result is the same as that obtained in the very near field of a thermal source when a microscopic length scale describing the non locality of the electromagnetic response is introduced [79]. The dependence of the degree of spatial coherence versus the lateral distance $\rho$ is plotted in Fig. 8 (red dashed line). Its width is on the order of $\ell_{c}$, corresponding to a spatial coherence length driven by the internal structure of the disordered system. The strong connection between the spatial variations of the near field and of the geometric structure of the medium producing this near field is a well known property, that is also a feature of the quasi-static regime [73]. The dependence of the degree of spatial coherence on $\ell_{c}$ gives a non-universal character to electromagnetic speckle correlations in the near field. Also note that the exact shape of the correlation function in this regime depends on the form of the correlator $\left\langle\delta \epsilon(\mathbf{r}) \delta \epsilon\left(\mathbf{r}^{\prime}\right)\right\rangle$. The result given here corresponds to a Gaussian correlated disorder as given in Eq. (27).

Non-universal statistics of near-field speckle patterns have also been derived in the case of surface scattering from a rough interface separating a homogeneous medium from a vacuum $[80,81]$. Based on a single-scattering perturbation theory, it was shown that the intensity-intensity spatial correlation function in the near field was linearly related to the spatial autocorrelation function of the surface profile.

\subsection{Measurement of near-field speckles}

\subsubsection{Near-field microscopy on disordered dielectrics}

Although the measurement of electromagnetic near fields is common for radiowaves and microwaves, it is a much more challenging issue in the optical regime. Indeed, since by "near fields" we mean fields recorded at distances from sources (or scatterers) much shorter than the wavelength, the length scales involved are typically in the range 10-100 $\mathrm{nm}$. In the last twenty years, Scanning Near-field Optical Microscopy (SNOM) has developed to become an established technique, permitting not only near-field imaging but also the manipulation of optical fields at subwavelength scales [74]. In SNOM, an image of the near field is produced by scanning a subwavelength tip along the surface. The intensity of the field scattered by the tip, and collected in free space by a microscope objective or through an optical fiber, produces the image with a resolution chiefly given by the tip size. Measuring a near-field speckle amounts to recording the near field scattered by a disordered sample. SNOM studies of near-field speckles have been reported in Refs. [82-85]. An example of far-field and near-field speckle produced by a strongly scattering material is shown in Fig. 9. In the near-field image recorded at a distance $z \ll \lambda$ (Fig. 9a), the typical scale of the speckle is reduced compared to that in the far-field image recorded at a distance $z \gg \lambda$ (Fig. 9b). The subwavelength spatial variations are only visible in the near-field image since they are associated to evanescent components of the angular spectrum and are filtered out upon propagation [73,74].

\subsubsection{Speckle spot size and spatial coherence length}

The typical length scale in the speckle pattern (speckle spot size) can be quantitatively defined as the width of the intensity-intensity spatial correlation function $\left\langle I(\mathbf{r}) I\left(\mathbf{r}^{\prime}\right)\right\rangle$, that is computed in practice as the autocorrelation of an image as that in Fig. 9. The variation of the speckle spot size versus the distance $z$ to the surface of the sample is shown in Fig. 10. 

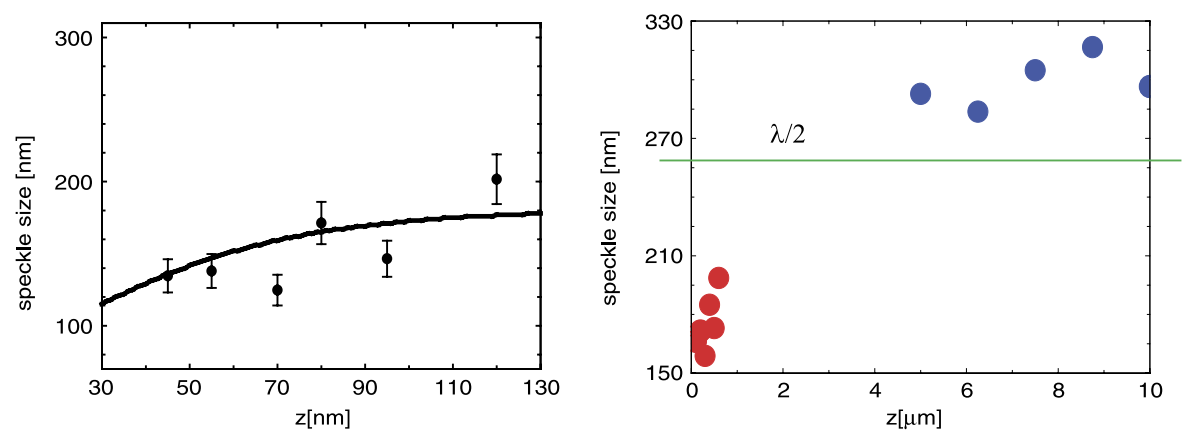

Fig. 10. Speckle spot size versus the distance $z$ to the surface. Same sample and illumination/detection conditions as in Fig. 9. The speckle spot size is estimated with data corrected for the point-spread function of the probe.

Source: Adapted from Ref. [83].

The data demonstrate a reduction of the speckle spot size when approaching the surface at subwavelength distance. Similar data have been reported with different samples in Ref. [82].

From the intensity-intensity spatial correlation function, one can also deduce the spatial coherence length, defined as the width of the degree of spatial coherence $\gamma_{E}\left(\mathbf{r}, \mathbf{r}^{\prime}\right)$ in Eq. (34). This can be done, at least qualitatively, by recalling that in a fully developed speckle, the complex amplitude of the field obeys circular Gaussian statistics (its real and imaginary parts are both Gaussian random variables). In that case, higher order correlation functions factorize into products of second-order correlations functions. Ignoring polarization issues in a qualitative analysis, one has $\left\langle I(\mathbf{r}) I\left(\mathbf{r}^{\prime}\right)\right\rangle=\left\langle E(\mathbf{r}) E^{*}(\mathbf{r}) E\left(\mathbf{r}^{\prime}\right) E^{*}\left(\mathbf{r}^{\prime}\right)\right\rangle \sim$ $\left|\gamma_{E}\left(\mathbf{r}, \mathbf{r}^{\prime}\right)\right|^{2}$. The dependence of the speckle spot size (spatial coherence length) on the distance $z$ to the surface is shown in Fig. 10. One clearly observes a decrease of the spatial coherence length in the near field, that reaches subwavelength values. Around $z=50 \mathrm{~nm}$, the dependence on $z$ is in qualitative agreement with the theoretically predicted linear dependence. Indeed, the calculated expression of the degree of spatial coherence in the quasi-static regime Eq. (59) predicts a spatial coherence length that scales with the distance $z$.

Near-field optical measurements in disordered scattering samples have also been reported in Ref. [85]. In this experiment, differences between far-field and near-field speckles have been observed unambiguously. But the analysis of the intensity-intensity spatial correlation function $\left\langle I(\mathbf{r}) I\left(\mathbf{r}^{\prime}\right)\right\rangle \sim\left|\gamma_{E}\left(\mathbf{r}, \mathbf{r}^{\prime}\right)\right|^{2}$ was incorrectly based on Eq. (45) for the degree of spatial coherence. This expression is not valid outside the medium, and does not account for the near-field components that dominate at subwavelength distance from the output surface.

\subsubsection{Other evidences of non-universality in near-field speckles}

Beyond the study of disordered dielectric materials, SNOM has been used to measure near-field speckles on other kinds of photonic materials. Spatial correlations in near-field speckle patterns measured on disordered photonic crystals have been shown to provide information about the incomplete band gap of such structures [86]. Disordered metallic films made of noble metals (silver or gold) are also known to produce subwavelength intensity fluctuations in the near field, due to the interplay between material resonances (surface plasmons) and multiple scattering in the complex geometry close to the electric percolation threshold [87]. For wavelengths in the near infrared, a resonant regime is observed that leads to large spatial intensity fluctuations. The intensity-intensity spatial correlation function has been measured in this regime, and shown to exhibit a specific lineshape that goes beyond that of an effective purely dielectric or metallic homogeneous material [88]. Another study on similar systems has shown major differences between the speckles produced in the resonant (near infrared) and non-resonant (visible) regimes [89]. In the non-resonant regime, the speckle can be understood as being produced by a rough surface in a weakly scattering regime, and the correlation function can be quantitatively described using the perturbation theory developed in Refs. $[80,81]$. In particular, the intensity-intensity spatial correlation function is linearly related to the spatial autocorrelation function of the surface profile, thus exhibiting a high degree of non-universality. Another study has shown differences between near-field speckles produced by an illumination in transmission through a thick sample in the multiple scattering regime, and by a local illumination in reflection on the same sample [84]. Deviations from the Rayleigh statistics for the intensity distribution have been observed, indicating that the hypotheses of a fully developed speckle could break down. As in the case of scattering from a rough surface of a homogeneous material, under a local illumination in reflection the near-field speckle can be dominated by surface contributions, also inducing a high degree of non universality.

\subsection{Correlations and fluctuations due to an internal source}

We have studied so far speckle patterns produced by external illumination on a strongly scattering medium and observed either deep inside the medium or in transmission through it. When the speckle pattern is produced by a localized source placed inside the medium, a different type of spatial correlations is generated. This issue was first examined in terms of spatial intensity-intensity correlations, leading to the so-called $C_{0}$ correlation [90]. This name was chosen since this new 
contribution to the intensity-intensity correlation function was an additional term to the $C_{1}, C_{2}$ and $C_{3}$ contributions known in mesoscopic physics [24,91]. The $C_{0}$ speckle correlation possesses two interesting features: It is of infinite range [90], and is non-universal [92], due to its sensitivity to the internal structure of the disordered medium. This dependence on the local environment is driven near-field interactions [93,94], as we shall see. This means that microscopic length scales, that characterize the internal structure of the scattering medium, are encoded in the $C_{0}$ contribution, making its measurement useful for sensing or imaging in complex media. We shall also see that the $C_{0}$ contribution to the spatial intensity-intensity correlation function of the speckle pattern equals the fluctuation of the local density of states (LDOS) at the source position, a remarkable result initially derived for scalar waves in Ref. [95], and revisited for electromagnetic waves in Ref. [93].

\subsubsection{Intensity-intensity correlation function}

Let us first establish a general expression of the intensity-intensity correlation function in a speckle pattern observed in the far field of a finite-size sample of disordered material. The sample is illuminated by a monochromatic electric-dipole point source with amplitude $\mathbf{p}$ oscillating at frequency $\omega$. The far-field intensity radiated in a given direction $\mathbf{u}$ per unit solid angle is

$$
I(\mathbf{u})=\lim _{r \rightarrow \infty} \frac{\epsilon_{0} c}{2} r^{2}|\mathbf{E}(\mathbf{r})|^{2}
$$

where $\mathbf{r}=r \mathbf{u}$ and $\mathbf{E}(\mathbf{r})$ is the electric field radiated by the dipole source. The variation of the intensity versus the angular variable $\mathbf{u}$ forms a speckle pattern. Its fluctuations can be characterized by the normalized angular correlation function $C\left(\mathbf{u}, \mathbf{u}^{\prime}\right)$ defined as

$$
C\left(\mathbf{u}, \mathbf{u}^{\prime}\right)=\frac{\left\langle\delta I(\mathbf{u}) \delta I\left(\mathbf{u}^{\prime}\right)\right\rangle}{\langle I(\mathbf{u})\rangle\left\langle I\left(\mathbf{u}^{\prime}\right)\right\rangle}=\frac{\left\langle I(\mathbf{u}) I\left(\mathbf{u}^{\prime}\right)\right\rangle}{\langle I(\mathbf{u})\rangle\left\langle I\left(\mathbf{u}^{\prime}\right)\right\rangle}-1
$$

where $\delta I(\mathbf{u})=I(\mathbf{u})-\langle I(\mathbf{u})\rangle$.

Under the condition of statistically isotropy of the speckle field, the angular intensity correlation function $C\left(\mathbf{u}, \mathbf{u}^{\prime}\right)$ is a function of $\cos \Theta=\mathbf{u} \cdot \mathbf{u}^{\prime}$. Moreover, defining the total emitted power $P$ by $P=\int_{4 \pi} I(\mathbf{u}) d \mathbf{u}$, where du means integration over the solid angle, statistical isotropy also implies that $\langle I(\mathbf{u})\rangle=\langle P\rangle /(4 \pi)$. This assumption is valid if the disordered medium is itself statistically isotropic, and if averaging over the orientation of the point dipole source is performed, two conditions that we assume in the following. Let us compute the normalized variance $\operatorname{Var}(P) /\langle P\rangle^{2}$ of the total emitted power. In terms of the angular intensity correlation function, it can be written

$$
\frac{\left\langle P^{2}\right\rangle-\langle P\rangle^{2}}{\langle P\rangle^{2}}=\frac{1}{16 \pi^{2}} \int_{4 \pi} \int_{4 \pi} C\left(\mathbf{u} \cdot \mathbf{u}^{\prime}\right) d \mathbf{u} d \mathbf{u}^{\prime} .
$$

The integral can be calculated by expanding the angular correlation function on the basis of Legendre polynomials in the form $C(\cos \Theta)=\sum_{n=0}^{\infty} a_{n} P_{n}(\cos \Theta)$. Using the addition theorem of spherical harmonics [96], we can write

$$
P_{n}(\cos \Theta)=P_{n}(\cos \theta) P_{n}\left(\cos \theta^{\prime}\right)+2 \sum_{m=1}^{n} \frac{(n-m) !}{(n+m) !} P_{n}^{m}(\cos \theta) P_{n}^{m}\left(\cos \theta^{\prime}\right) \cos \left[m\left(\phi-\phi^{\prime}\right)\right]
$$

where $(\theta, \phi)$ and $\left(\theta^{\prime}, \phi^{\prime}\right)$ are the polar and azimuthal angles in spherical coordinates corresponding to the directions $\mathbf{u}$ and $\mathbf{u}^{\prime}$, respectively, and $P_{n}^{m}(x)$ are the associated Legendre functions. Inserting this expansion into Eq. (63) and integrating over $\phi$ and $\phi^{\prime}$, we obtain

$$
\frac{\left\langle P^{2}\right\rangle-\langle P\rangle^{2}}{\langle P\rangle^{2}}=\frac{1}{4} \sum_{n=0}^{\infty} a_{n}\left[\int_{-1}^{+1} P_{n}(x) d x\right]^{2} .
$$

Since $P_{0}(x)=1$, the last integral is $\int_{-1}^{+1} P_{n}(x) d x=\int_{-1}^{+1} P_{n}(x) P_{0}(x) d x=2 \delta_{n_{0}}$ due the orthogonality condition $\int_{-1}^{+1} P_{n}(x)$ $P_{m}(x) d x=2 \delta_{n m} /(2 n+1)$ of Legendre polynomials, where $\delta_{n m}$ is the Kronecker delta. This leads to

$$
\frac{\left\langle P^{2}\right\rangle-\langle P\rangle^{2}}{\langle P\rangle^{2}}=a_{0} .
$$

This equality between the variance of the total emitted power and the constant term $a_{0}$ in the angular intensity correlation function is the essence of the $C_{0}$ speckle correlation $[90,93,95]$. For consistency with the usual notations in mesoscopic physics we will write $a_{0}=C_{0}$. The full intensity-intensity angular correlation function can now be rewritten as $C\left(\mathbf{u} \cdot \mathbf{u}^{\prime}\right)=$ $C_{0}+C^{\prime}\left(\mathbf{u} \cdot \mathbf{u}^{\prime}\right)$. The $C^{\prime}\left(\mathbf{u} \cdot \mathbf{u}^{\prime}\right)$ contribution, defined by this relation, is the usual angular correlation function that is observed in speckles produced by an external illumination (for example by a laser beam), with a source placed at infinity. The LDOS fluctuations at the source position are negligible in this case, and the $C_{0}$ contribution vanishes. It has been shown that $C^{\prime}\left(\mathbf{u} \cdot \mathbf{u}^{\prime}\right)$ contains three classes of contributions, historically denoted by $C_{1}, C_{2}$ and $C_{3}$ [91]. This splitting has been widely used in mesoscopic physics [24]. The $C_{1}$ term corresponds to the approximation of a fully developed speckle pattern with a 
field obeying Gaussian statistics, and the terms $C_{2}$ and $C_{3}$ are non-Gaussian corrections (in addition to the $C_{0}$ contribution that is also non-Gaussian). In order to comply with the standard way of writing the expansion of the intensity-intensity correlation function, we can write:

$$
C\left(\mathbf{u} \cdot \mathbf{u}^{\prime}\right)=C_{0}+C_{1}\left(\mathbf{u} \cdot \mathbf{u}^{\prime}\right)+C_{2-3}\left(\mathbf{u} \cdot \mathbf{u}^{\prime}\right)
$$

where

$$
C_{0}=\frac{\left\langle P^{2}\right\rangle-\langle P\rangle^{2}}{\langle P\rangle^{2}}
$$

and $C_{2-3}\left(\mathbf{u} \cdot \mathbf{u}^{\prime}\right)$ contains all non-Gaussian corrections beyond $C_{0}$ (we do not discuss here the splitting of this term into $C_{2}$ and $C_{3}$ contributions). It can be established from multiple scattering theory that in the limit $k_{0} \ell_{s} \gg 1$, the $C_{2-3}$ contribution is negligible compared to $C_{1}[24,91]$. Eq. (67) is the most general expansion of the angular intensity-intensity correlation function due to an internal point source, valid under the only assumption of statistical isotropy. The $C_{1}$ term, that corresponds to the Gaussian field approximation, can be factorized and written in terms of the field-field correlation function [97]:

$$
C_{1}\left(\mathbf{u} \cdot \mathbf{u}^{\prime}\right)=\left|\frac{\sum_{k}\left\langle E_{k}(\mathbf{r}) E_{k}^{*}\left(\mathbf{r}^{\prime}\right)\right\rangle}{\left\langle|\mathbf{E}(\mathbf{r})|^{2}\right\rangle}\right|^{2}=\left|\gamma_{E}\left(\mathbf{r}, \mathbf{r}^{\prime}\right)\right|^{2}
$$

where $\mathbf{E}(\mathbf{r})$ and $\mathbf{E}\left(\mathbf{r}^{\prime}\right)$ are far-field amplitude along direction $\mathbf{u}$ and $\mathbf{u}^{\prime}$, and $\gamma_{E}\left(\mathbf{r}, \mathbf{r}^{\prime}\right)$ is the degree of spatial coherence defined in Eq. (34).

\subsection{2. $C_{0}$ correlation and fluctuations of the local density of states}

For a dipole source placed at position $\mathbf{r}_{s}$ inside the medium, the total emitted power $P$ is $P=(\omega / 2) \operatorname{Im}\left[\mathbf{p}^{*} \cdot \mathbf{E}\left(\mathbf{r}_{s}\right)\right]$, where $\mathbf{E}\left(\mathbf{r}_{s}\right)$ is the electric field at the source position. Using the dyadic Green function $\mathbf{G}\left(\mathbf{r}, \mathbf{r}^{\prime}, \omega\right)$ of the disordered medium, it can be rewritten as

$$
P=\frac{\mu_{0} \omega^{3}}{2} \operatorname{Im}\left[\mathbf{p}^{*} \cdot \mathbf{G}\left(\mathbf{r}_{s}, \mathbf{r}_{s}, \omega\right) \mathbf{p}\right] .
$$

The emitted power averaged over the orientations of the electric dipole $\mathbf{p}$ is

$$
P=\frac{\mu_{0} \omega^{3}}{6}|\mathbf{p}|^{2} \operatorname{Im}\left[\operatorname{Tr} \mathbf{G}\left(\mathbf{r}_{s}, \mathbf{r}_{s}, \omega\right)\right]
$$

Introducing the local density of states (LDOS) defined as [98,43]:

$$
\rho\left(\mathbf{r}_{s}, \omega\right)=2 \omega /\left(\pi c^{2}\right) \operatorname{Im}\left[\operatorname{Tr} \mathbf{G}\left(\mathbf{r}_{s}, \mathbf{r}_{s}, \omega\right)\right]
$$

the emitted power takes the form

$$
P=\pi \omega^{2} /\left(12 \epsilon_{0}\right)|\mathbf{p}|^{2} \rho\left(\mathbf{r}_{s}, \omega\right) .
$$

Inserting (73) into (68), we directly obtain

$$
C_{0}=\frac{\left\langle\rho^{2}\left(\mathbf{r}_{s}, \omega\right)\right\rangle}{\left\langle\rho\left(\mathbf{r}_{s}, \omega\right)\right\rangle^{2}}-1=\frac{\operatorname{Var}\left[\rho^{2}\left(\mathbf{r}_{s}, \omega\right)\right]}{\left\langle\rho\left(\mathbf{r}_{s}, \omega\right)\right\rangle^{2}} .
$$

This simple relationship shows that the constant term $C_{0}$ in the angular intensity-intensity correlation function equals the normalized variance of the LDOS at the source position. This result was first derived in Ref. [95] using a diagrammatic approach, and generalized in the present form in Ref. [93]. The derivation given here relies only on the assumptions of energy conservation and statistical isotropy of the medium. Therefore the result is valid in all wave transport regimes, including Anderson localization. Physically, the equality described by Eq. (74) can be understood in simple terms. For a point dipole source with given amplitude $\mathbf{p}$, changes in the LDOS correspond to changes in the power transferred to the environment. In a non absorbing medium, this power is radiated in the far field, producing the angular speckle pattern. Therefore, LDOS fluctuations are transformed into global fluctuations of the speckle patterns, which themselves are encoded into a constant contribution to the angular correlation.

The equality between LDOS fluctuations and $C_{0}$ speckle correlations has practical consequences. In optics, LDOS fluctuations can be measured from the fluorescence lifetime of nanoscopic emitters [94,99-103], since the spontaneous decay rate (inverse of lifetime) is proportional to the LDOS in the weak-coupling regime. The full statistical distribution of the LDOS can even be measured (and not only its variance). In mesoscopic physics, the shape of the LDOS distribution is expected to carry specific signatures of the photon transport regime [104-107]. An important feature of LDOS fluctuations (or equivalently $C_{0}$ correlation) produced by electromagnetic sources is that the near-field interaction between the source and its local environment confers a non-universal character. Indeed, LDOS fluctuations can be extremely sensitive to the microscopic structure of the disordered medium $[94,108,109]$. They carry information on length scales beyond the scattering mean free path, an important property for sensing or imaging in complex media. 


\subsubsection{Single-scattering regime}

The non-universality of LDOS fluctuations can be demonstrated using an explicit calculation in the single-scattering regime, as initially shown in Refs. $[108,109]$. As a model of a disordered medium, we consider a spherical region with radius $R$, filled with individual particles with radius $a \ll \lambda$, where $\lambda$ is the emission wavelength. The particles are randomly placed within the sphere of radius $R$, forming a disordered cluster, and we assume negligible correlations between the positions of the particle. The electric-dipole source is placed at position $\mathbf{r}_{s}$, coinciding with the center of the cluster, and is surrounded by a spherical exclusion volume with radius $R_{0}$. This length scale $R_{0}$ is a microscopic length scale that characterizes the local environment of the source.

In the single-scattering regime, and for uncorrelated particle positions, the averages and standard deviations for a medium containing $N$ particles can be deduced from one-particle calculations. Indeed, one has $\langle\Gamma\rangle_{(N)}=N\langle\Gamma\rangle_{(1)}$ and $\sigma_{(N)}^{2}(\Gamma)=$ $N \sigma_{(1)}^{2}(\Gamma)$, where the subscripts $(N)$ and (1) refer to $N$-particle and one-particle quantities, respectively. The one-particle quantities can be calculated analytically, using the formalism of Ref. [110]. A particle at position $\mathbf{r}_{p}$ is described by its electric dynamic polarizability $\alpha(\omega)$, that includes radiation reaction [110,111]:

$$
\alpha(\omega)=\frac{\alpha_{0}(\omega)}{1-i\left(\omega^{3} / 6 \pi c^{3}\right) \alpha_{0}(\omega)}
$$

where $\alpha_{0}(\omega)$ is the quasi-static polarizability

$$
\alpha_{0}(\omega)=4 \pi a^{3} \frac{\epsilon(\omega)-1}{\epsilon(\omega)+2}
$$

and $\epsilon(\omega)$ is the dielectric function of the material. The Green function of the one-particle system, connecting two arbitrary points $\mathbf{r}$ and $\mathbf{r}^{\prime}$, can be written explicitly in the form

$$
\mathbf{G}\left(\mathbf{r}, \mathbf{r}^{\prime}, \omega\right)=\mathbf{G}_{0}\left(\mathbf{r}, \mathbf{r}^{\prime}, \omega\right)+\mathbf{G}_{0}\left(\mathbf{r}, \mathbf{r}_{p}, \omega\right) \cdot \alpha(\omega) \epsilon_{0} \mathbf{G}_{0}\left(\mathbf{r}_{p}, \mathbf{r}^{\prime}, \omega\right)
$$

where $\mathbf{G}_{0}\left(\mathbf{r}, \mathbf{r}^{\prime}, \omega\right)$ is the vacuum Green function given by

$$
\mathbf{G}_{0}\left(\mathbf{r}, \mathbf{r}^{\prime}, \omega\right)=\left[\mathbf{I}+\frac{1}{k_{0}^{2}} \nabla \nabla\right] \frac{\exp \left(i k_{0} \rho\right)}{4 \pi \rho} \text { for } \rho \neq 0
$$

with $\rho=\left|\mathbf{r}-\mathbf{r}^{\prime}\right|$.

Let us examine the limit of a small system satisfying $k_{0} R_{0} \ll k_{0} R \ll 1$. In this limit, the Green function can be expanded in terms of the small parameter $k_{0} r$, with $r=\left|\mathbf{r}_{p}-\mathbf{r}_{s}\right|$ the distance between the source and the particle. Simple explicit expressions can be obtained by keeping the leading terms in the calculation of the LDOS $\rho\left(\mathbf{r}_{s}, \omega\right)$ based on Eqs. (72) and (77) [108,109]. Using the simplified notations $\rho=\rho\left(\mathbf{r}_{s}, \omega\right)$ and $\rho_{0}=\rho_{0}(\omega)$, we obtain for the averaged value [112]:

$$
\left\langle\rho / \rho_{0}\right\rangle=1+\frac{11}{5} f \operatorname{Re}(\beta)\left(k_{0} R\right)^{2}+\frac{3 f}{\left(k_{0} R_{0}\right)^{3}} \operatorname{Im}(\beta)+2 f\left(\frac{a}{R_{0}}\right)^{3}|\beta|^{2}
$$

where $\beta(\omega)=[\epsilon(\omega)-1] /[\epsilon(\omega)+2]$ and $f$ is the volume fraction of the disordered medium. Note that for a non-absorbing medium, the dielectric function is real and the terms proportional to $\operatorname{Im}(\beta)$ vanish. As pointed out in Ref. [108], the different terms in the expression of the averaged normalized LDOS have different physical origins. In absence of absorption, the LDOS is proportional to the averaged power emitted in the far field. The radiated field is the sum of an averaged field and a fluctuating field, which we write as $\mathbf{E}=\langle\mathbf{E}\rangle+\delta \mathbf{E}$. The averaged emitted power $P$ contains two contributions: $P \propto|\langle\mathbf{E}\rangle|^{2}+\left\langle|\delta \mathbf{E}|^{2}\right\rangle$. The calculation leading to Eq. (79) shows that the term involving the cluster size $R$ arises from the power of the averaged field $|\langle\mathbf{E}\rangle|^{2}$. The last two terms, involving the microscopic scales $a$ and $R_{0}$, come from the power of the fluctuations $\left\langle|\delta \mathbf{E}|^{2}\right\rangle$ (in the presence of absorption, the leading term is the absorption term proportional to $\operatorname{Im}(\beta)$ that also involves the microscopic scale $R_{0}$ ).

The variance of the normalized LDOS, which drives the $C_{0}$ correlation discussed in this section, can be obtained in a similar way. It reads

$$
\begin{aligned}
\operatorname{Var}\left(\rho / \rho_{0}\right)= & f\left(k_{0} a\right)^{3}\left\{\frac{121}{75} k_{0} R[\operatorname{Re}(\beta)]^{2}+\frac{3}{\left(k_{0} R_{0}\right)^{9}}\left[\operatorname{Im}(\beta)+\frac{2}{3}\left(k_{0} a\right)^{3}|\beta|^{2}\right]^{2}\right. \\
& \left.+\frac{33}{5} \frac{\operatorname{Re}(\beta)}{\left(k_{0} R_{0}\right)^{4}}\left[\operatorname{Im}(\beta)+\frac{2}{3}\left(k_{0} a\right)^{3}|\beta|^{2}\right]\right\} .
\end{aligned}
$$

Here also terms involving the cluster size $R$ and terms involving the microscopic scale $R_{0}$ are easily identified. Finite-size effects are responsible for the dependence on $R$. Interestingly, the dependence on $R_{0}$ confers to LDOS fluctuations a nonuniversal behavior. By measuring long-range intensity-intensity correlations in the far-field speckle pattern produced by an internal point source, or equivalently LDOS fluctuations, one can get a signature of microscopic length scales in the disordered medium. 


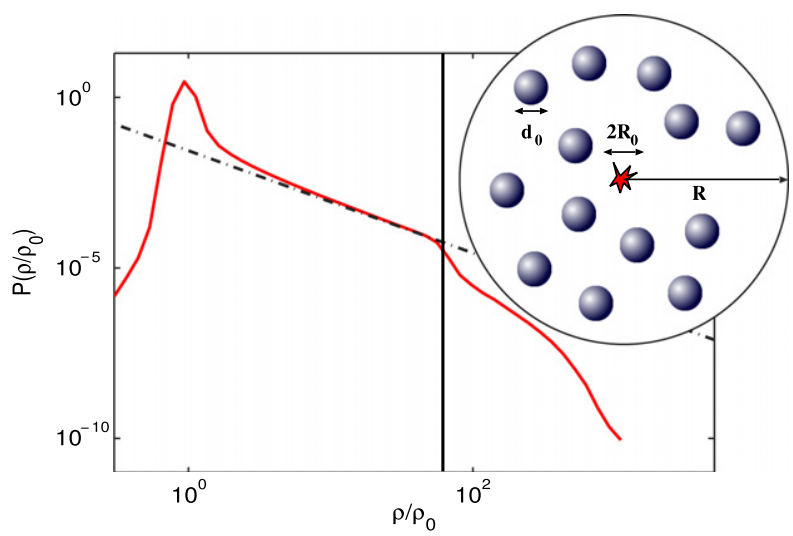

Fig. 11. Statistical distribution of the normalized LDOS $\rho\left(\mathbf{r}_{S}, \omega\right) / \rho_{0}(\omega)$ at a wavelength $\lambda=0.63 \mu$ m obtained from numerical simulations. The medium is a three-dimensional cluster of nanoparticle (behaving as electric dipoles), randomly distributed within a spherical cluster with radius $R=1.2 \mu$ m. The exclusion radius around the source is $R_{0}=0.05 \mu \mathrm{m}$. The scattering mean free path is $\ell_{s}=1.9 \mu \mathrm{m}\left(k_{0} \ell_{s}=19\right.$ and $\left.R / \ell_{s}=0.63\right)$. Inset: Schematic view of the system.

Source: From Ref. [93].

\subsubsection{Evidence of near-field interactions and non universality}

The limit $k r \ll 1$ used in the single-scattering model is the quasi-static limit in which near-field interactions between the source and the particle dominate. In this regime, well-known in near-field optics [73], the electric-dipole field is dominated by a contribution scaling as $1 / r^{3}$, that is responsible for the sensitivity of the LDOS to the presence of a particle at subwavelength distance [110]. The dependence on the microscopic length scale $R_{0}$ in Eq. (80) is a direct consequence of the near-field interactions between the source at $\mathbf{r}_{s}$ and the nearby particles $\left(R_{0}\right.$ can be understood as the minimum distance between the source at $\mathbf{r}_{s}$ and the nearest particle). This means that the non-universality of LDOS fluctuations, or $C_{0}$ speckle correlations, is essentially a near-field effect.

Statistical distributions of the LDOS in scattering media, and the sensitivity of LDOS fluctuations to the microscopic internal structure, can be studied on the basis of numerical simulations. An example of a numerical result is shown in Fig. 11. The LDOS at the center $\mathbf{r}_{s}$ of a disordered cluster of nanoparticles (modeled as electric dipoles) is calculated by solving Maxwell's equations in three dimensions without any approximations $[93,108,109]$. Calculations are made for a large number of configurations (random positions of the nanoparticles), allowing one to build up the full statistical distribution of the normalized LDOS $\rho / \rho_{0}$. The wide and asymmetric lineshape of the distribution has been discussed in detail in Refs. [108,109]. Here we focus on the region for which $\rho / \rho_{0}>1$.

A power law decay with exponent $(-3 / 2)$ is observed for $\rho / \rho_{0} \simeq 10$, indicated by the dashed line in Fig. 11 . This is the signature of near-field interaction with the nearest particle. Indeed, for $r=\left|\mathbf{r}_{p}-\mathbf{r}_{s}\right| \sim R_{0}$, one finds that in the quasi-static limit

$$
\frac{\rho-\rho_{0}}{\rho_{0}} \sim 2 \beta^{2}\left(\frac{a}{r}\right)^{6}
$$

The probability density of the radial position of the particle is $P\left(k_{0} r\right) \sim\left(k_{0} r\right)^{2}$. A change of variable using Eq. (81) leads to a probability density of the $\operatorname{LDOS} P\left(\rho / \rho_{0}\right) \sim\left(\rho / \rho_{0}\right)^{-3 / 2}$, thus explaining the power law decay in simple terms.

The single scattering cut-off (indicated by the vertical solid line in Fig. 11), corresponds to the largest possible value of $\rho / \rho_{0}$ in this regime, obtained for $r=R_{0}$. Beyond this cut-off, larger values of $\rho / \rho_{0}$ are due to near-field interactions with more than one scatterer. This makes this part of the distribution sensitive to the local correlation in the positions of the particle [93]. Since the variance of the distribution is sensitive to the exact shape of the cut-off in the lineshape, LDOS fluctuations (or the $C_{0}$ correlation) is sensitive to the microscopic structure of the disordered medium around the source point $\mathbf{r}_{s}$. This confirms the non-universal character discussed previously based on the single-scattering model.

The crucial role of the microscopic structure can be made even more striking by computing LDOS distributions in systems with the same scattering mean free path $\ell_{s}$ [i.e. with the same scattering strength $1 /\left(k_{0} \ell_{s}\right)$ ] but different microscopic length scale $R_{0}$. We show in Fig. 12 the fluctuations of the LDOS in disordered clusters with the same value of $k_{0} \ell_{s}$, but with $R_{0}=0.07 \mu \mathrm{m}$ or $R_{0}=0.14 \mu \mathrm{m}$. The simulation clearly shows that $k_{0} \ell_{s}$ cannot be considered as the single parameter driving the statistical distribution, and therefore LDOS fluctuations or the $C_{0}$ correlation. Moreover, systems with the same exclusion radius $R_{0}$ give rise to similar values of $C_{0}$ (the computed values of $C_{0}$ for each curve are shown in the inset) and similar statistical lineshapes, even with different densities of scatterers. This confirms the sensitivity of LDOS fluctuations, or $C_{0}$ correlation, to the local environment of the source.

Statistical distributions of LDOS in strongly scattering materials can also be measured experimentally at optical wavelength. An approach consists in dispersing fluorescent nanosources (e.g. fluorescent nanobeads, dye molecules or fluorescent nanocrystals) inside or on top of a scattering medium [94,100-102]. Experiments in non-absorbing volumescattering materials, that mimic the model systems studied theoretically, use powders made of a high index material (such a $\mathrm{ZnO}$ at wavelength $\lambda \sim 600-700 \mathrm{~nm}$ ). An example of measured LDOS distributions by Sapienza et al. [94] is shown in Fig. 13. 


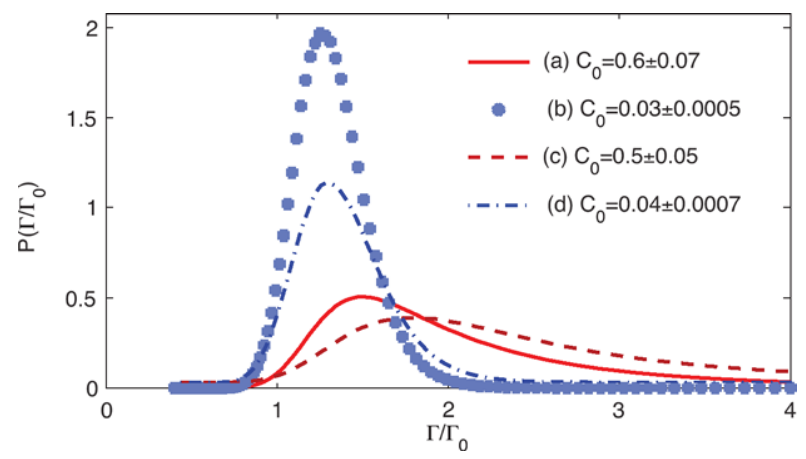

Fig. 12. Numerical simulation of the statistical distribution of the normalized decay rate $\Gamma / \Gamma_{0}=\rho / \rho_{0}[112]$ of a dipole emitter placed at the center $\mathbf{r}_{S}$ of a disordered cluster similar to that in Fig. 11. Four different systems are considered, with the same value of $k_{0} \ell_{s}=9.4$ and $R=0.63 \mu \mathrm{m}$. Curves (a) and (c) correspond to an exclusion radius $R_{0}=0.07 \mu \mathrm{m}$ while curves (b) and (d) correspond to an exclusion radius $R_{0}=0.14 \mu \mathrm{m}$. Curves (c) and (d) are the same as (a) and (b) but with a doubled density of scatterers. Source: From Ref. [94].

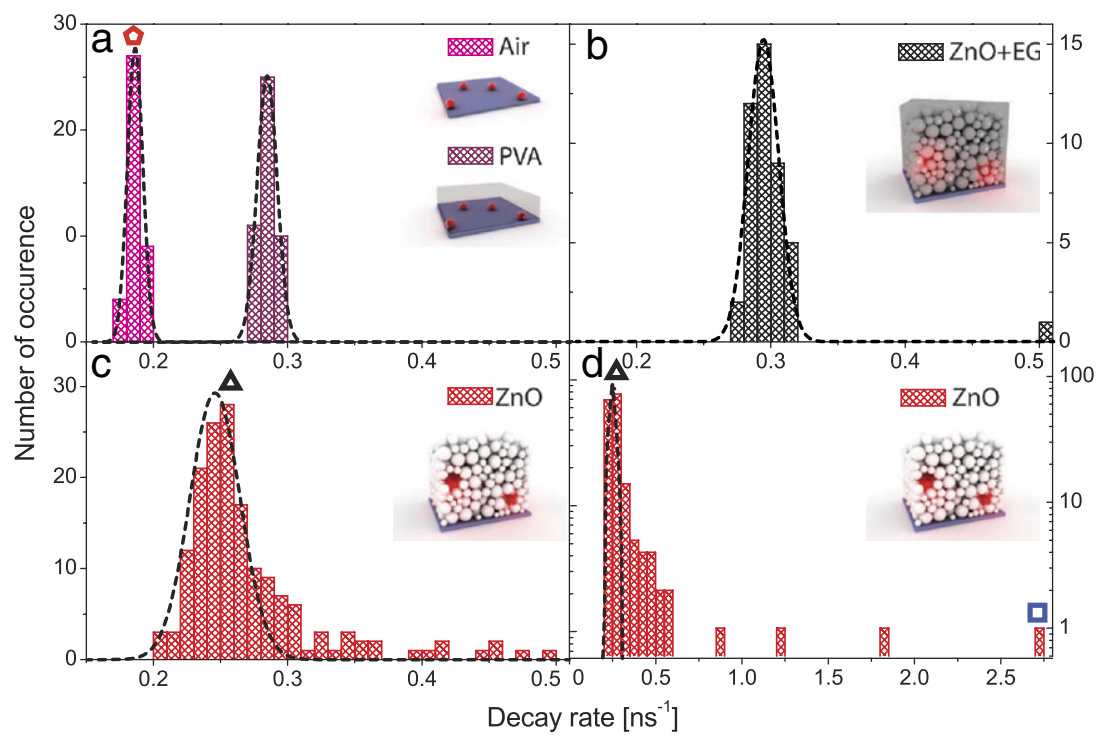

Fig. 13. Measured statistical distributions of the spontaneous decay rate $\Gamma \propto \rho\left(\mathbf{r}_{s}, \omega\right)$ of fluorescent beads (nanosources with $20 \mathrm{~nm}$ diameter) in different environments. (a) Beads on a glass-air and glass-PVA interface (reference). (b) Beads in a non-scattering ZnO + EG sample (EG suppresses the refractive index contrast). (c-d) Beads in the strongly scattering $\mathrm{ZnO}$ powder (transport mean free path $\ell=0.9 \mu \mathrm{m}$ ). (d) is a zoom out of (c) in log scale that shows the asymmetric tail and several extremely high decay rates. Source: From Ref. [94].

These measurements demonstrate the asymmetric shape of the LDOS distribution (inferred from the distribution of the decay rate $\Gamma \propto \rho\left(\mathbf{r}_{s}, \omega\right)$ of fluorescent beads, Fig. 13c), and the existence of large values of $\rho / \rho_{0}$ driven by near-field interactions. This was the first experimental evidence of the sensitivity of LDOS fluctuations to the local environment in a volume scattering material in the multiple scattering regime.

\subsection{Polarization length scales}

In general, material properties are scale dependent, and one can define different regimes of interaction with electromagnetic fields. A microscopic regime refers to length scales over which the material is homogeneous and the emerging field is usually fully polarized (by "microscopic" here we refer to scales smaller than the material heterogeneities, but much larger than atomic sizes so that the description of matter at the continuous level still holds). At the largest macroscopic scale, the optical characteristics involve significant averaging over volumes much larger than the characteristic scales of inhomogeneities and, as a result, only effective properties can be recovered. Thus, a microscopic description provides the intrinsic dielectric properties of the constituents, while a description at macroscopic scales implies an effective averaging of the dielectric properties. However, at intermediate mesoscopic scales, fluctuations around the average become important, and may provide different information about structural morphology.

For large scale randomly inhomogeneous media, a multiscale description of the light propagation can be envisioned where Maxwell's equations, the radiative transfer or diffusion equations are applied to describe the microscopic, mesoscopic, 

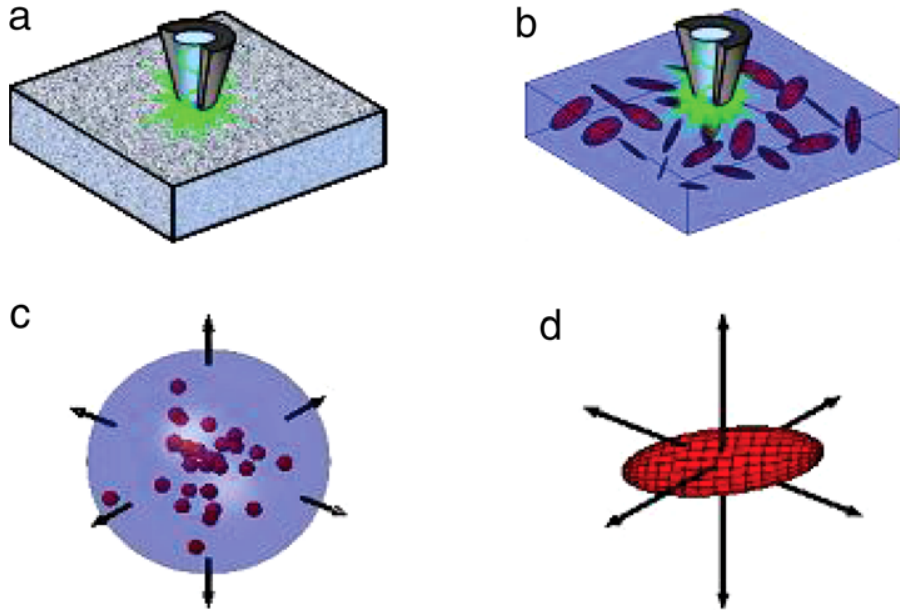

Fig. 14. (a) An inhomogeneous material system probed at mesoscopic scales through a local excitation provided by a near-field optical probe. (b) The observed far field response of the material system may be interpreted as being determined by a discrete ensemble of anisotropic scatterers with different local polarizabilities and orientations. (c) An inhomogeneous material system where the physical dimensions of the object limit the extents of the field material interaction is equivalent to an effective anisotropic scatterer (d).

Source: From Ref. [114].

and macroscopic scales, respectively. It should be anticipated that the observed optical response depends on the volume probed experimentally as schematically depicted in Fig. 14. This concept has been used, for instance, to describe different statistical regimes in near-field scattering from random media [113]. At such intermediate scales, one has to go beyond conventional effective medium approaches, which are based on assigning some effective dielectric permittivity to the local properties. In the case of an isotropic distribution of optically isotropic constituents, the effective dielectric permittivity is scalar. However, if the volume of averaging is limited, the local properties can no longer be described with a scalar permittivity and, as suggested in Fig. 14, one has to locally assign an effective dielectric polarizability tensor. Its components and orientation may depend on the particular location as suggested in Fig. 14(b) or may change from one mesoscopic object to another as illustrated in Fig. 14(d).

In a scattering experiment, the relationship between a real polarizability $\alpha$ and the fully polarized incident and scattered fields is commonly described in terms of the corresponding cross-spectral density matrices as $\mathbf{W}_{s c}=\mathbf{H}^{T} \alpha \mathbf{W}_{\text {inc }} \alpha \mathbf{H}$, where $\mathbf{W}_{s c}$ and $\mathbf{W}_{\text {inc }}$ are the cross-spectral density matrices of the scattered and excitation fields, $\mathbf{H}$ accounts for the field propagation to the detector, and the superscript $T$ stands for transpose. In general, for a fixed orientation of an effective polarizability, there are six unknown elements in the symmetric polarizability tensor. They can be recovered using a scheme based on three independent excitation fields along with a polarimetric detection of scattered intensities. If the polarizability remains constant during a set of observations, and only the orientation of the effective anisotropic polarizability changes randomly, one can apply an inversion method such as stochastic scattering polarimetry (SSP) $[115,116]$ to recover an anisotropic polarizability even without knowing the incident state of polarization. In situations where the magnitude of the polarizability varies, a sequential excitation procedure has to be implemented in order to recover the polarizability tensor. An eigenvalue decomposition followed by an ordering procedure can then be used to find the diagonal elements $\alpha_{a}, \alpha_{b}, \alpha_{c}$ of the polarizability tensor. An example is illustrated in Fig. 15. The two media examined in Fig. 15 are simulated based on a hard sphere model of the inclusion packing without any preferential orientation of the effective polarizability $[117,118]$. However, the values found for the tensor elements clearly show that, at this mesoscopic scale, the polarizabilities are not only anisotropic but they are also different for the two inhomogeneous materials as clearly seen. For medium (a), the smaller dielectric contrast between the host and the inclusions leads to a narrower distribution of the diagonal elements of the polarizability tensor and, consequently, to smaller fluctuations in the scattered fields. In the case of medium (b), however, the larger dielectric contrast results in a larger separation between the values of $\alpha_{a}, \alpha_{b}, \alpha_{c}$ which, in turn, generates even larger intensity variations.

Furthermore, a local anisotropic polarizability (LAP) can be quantified by a so-called anisotropy factor [119,120], defined as the contrast calculated for diagonal components of polarizability tensor

$$
\Delta=\frac{\sqrt{3 \operatorname{Tr}\left(\alpha^{2}\right)-\operatorname{Tr}(\alpha)^{2}}}{\operatorname{Tr}(\alpha)}=\sqrt{2} \frac{\sqrt{\alpha_{a}\left(\alpha_{a}-\alpha_{b}\right)+\alpha_{b}\left(\alpha_{b}-\alpha_{c}\right)+\alpha_{c}\left(\alpha_{c}-\alpha_{a}\right)}}{\alpha_{a}+\alpha_{b}+\alpha_{c}}
$$

where $\operatorname{Tr}$ denotes the trace of a tensor. The anisotropy factor in Eq. (82) allows now to examine the LAP's dependence on the volume of interaction. In doing so, we find that $\Delta$ always attains a maximum as can be seen in Fig. 16. This maximum anisotropy length (MAL) defines a new length scale characterizing the interaction between electromagnetic fields and complex materials. It represents the length scale over which the response of inhomogeneous medium is most sensitive to the polarization (vector) properties of the excitation field. In other words, it is at this scale that, on average, light depolarizes 

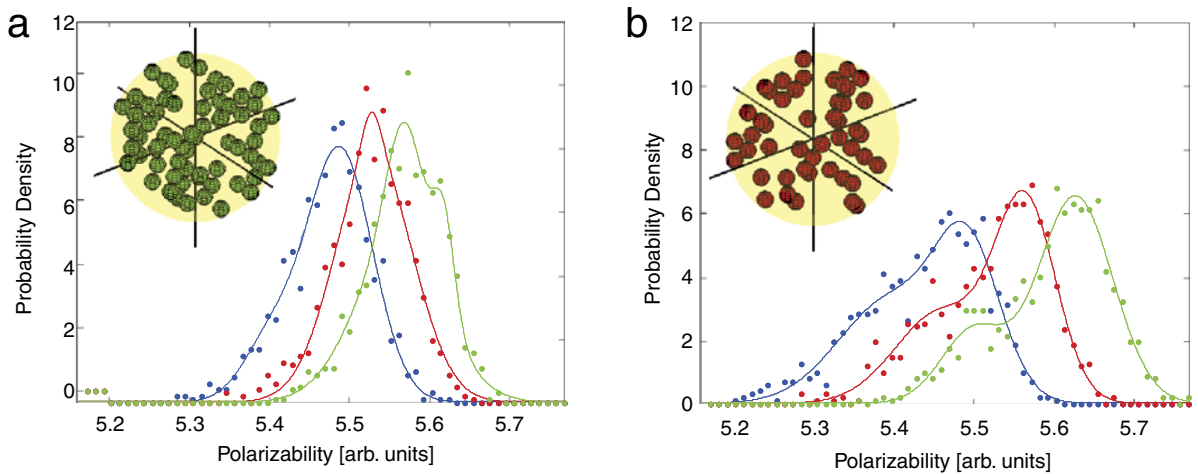

Fig. 15. Probability density functions of diagonal elements of the effective polarizability tensor. The volume of interaction corresponds to a sphere of 50 $\mathrm{nm}$ in diameter having a host refractive index of 1.33 and (a) inclusions with refractive index of 2 and a volume fraction of 0.27 and (b) inclusions with refractive index of 2.4 and a volume fraction of 0.18 . The dots are the results of the direct numerical calculations while the solid lines are best fits of the data collected in numerical scattering experiments.

Source: From Ref [114].

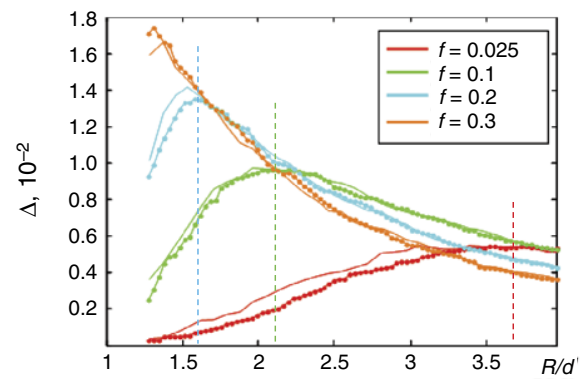

Fig. 16. Effective anisotropy factor $\Delta$ as a function of excitation volume $R$ normalized by inclusion diameter $d$ for spherical inclusions with a refractive index of 1.5 randomly distributed in vacuum. The continuous lines correspond to inclusions with diameter $\lambda / 32$ while the dotted lines correspond to inclusions of diameter $\lambda / 64$. The dotted lines indicate the corresponding MAL values.

Source: Adapted from Ref. [114]

most efficiently. Along with scattering mean free path and transport mean free path that describe the way the energy is transferred, MAL represents another length scale that characterizes the interaction between polarized fields and disordered media. As seen in Fig. 16, because of the spherical symmetry of individual scattering centers, the local polarizability is isotropic at length scales smaller than MAL. At larger scales, the macroscopic behavior is gradually approached which is characterized, again, by an effectively isotropic scattering volume with $\Delta=0$. At intermediate scales, the highest sensitivity to polarization determines corresponding values of MAL. We note that the anisotropy factors in Fig. 16 appear to be independent of the size of inhomogeneities. This happens because the interaction inside the inhomogeneous volume was primarily in the electrostatic regime. Also noticeable is the faster decay of $\Delta$ for higher number of inclusions inside the volume of interaction indicating that a larger number of scatterers within the excitation volume represents in fact a more isotropic medium.

The properties of the local anisotropic polarizability and the scale over which it becomes isotropic depend on the detailed morphology of the inhomogeneous medium and are, therefore, nonuniversal. This new scale length can be probed experimentally and provides information about the local structure of inhomogeneous media that otherwise may appear similar.

\section{Conclusion}

In closing we would like to remind that, traditionally, statistical optics has been guided by the overarching consequences of the central limit theorem, which led to the discovery of a number of universal properties of random electromagnetic fields, either created by fluctuating sources (coherence theory) or by scattering in disordered materials (speckle). Various phenomena and the associated observables are usually discussed in terms of independent and identically distributed random variables obeying convenient Gaussian distributions. However, in practice, the asymptotic properties deriving from all these constraints are approximations that in some instances are too restrictive.

Non-Gaussian behavior prevails in many experiments and the identity of real-life situations is most of the time preserved. Nevertheless, describing and using this non-universality requires new measures and estimates, often developed in the specific context of the random light-matter interaction that gives rise to such non-Gaussian properties. Here we surveyed recent developments aimed at characterizing higher-order, non-local and non-universal properties of three-dimensional speckle fields. 
The second-order statistics of random electromagnetic fields in the frequency domain is described by the cross-spectral density matrix W. Quantitative measures of spatial coherence and polarization properties can be directly defined from $\mathbf{W}$, sometimes through some degrees aimed at describing the coherence-like or polarization-like properties, or both. But one has to keep in mind that no additional information can be obtained that is not initially included in $\mathbf{W}$, and that any measure should be connected to measurable quantities in some interferometric type of experiment. The complex degree of mutual polarization (CDMP) quantifies the similarity between the polarization states at different locations. The degree of spatial coherence $\gamma_{E}$ describes spatial coherence and averages out the polarization properties. In a speckle field that is statistically homogeneous and isotropic, it is given by the cross density of states (CDOS), that measures the number of electromagnetic eigenmodes connecting two different points. The CDOS can be understood as a measure of the intrinsic spatial coherence of the field scattered by the medium, independently on the illumination conditions. Its width defines an intrinsic spatial coherence length, that depends on the wavelength but also on microscopic length scales of the disordered medium. In general, these measures have explicit spectral characteristics that relate to the source of random electromagnetic fields (partially coherent primary sources or secondary sources produced by scattering in a disordered medium).

When electromagnetic fields are randomized as a result of interaction with matter, their non-universal properties are determined by the internal structure of the material systems. As a result, specific microscopic scales of interaction influence the characteristics of measurable properties. The microscopic structure of the disordered medium influences the degree of spatial coherence in a speckle measured at short distance from the surface due to the strong influence of evanescent near fields. It also affects the magnitude of the intensity-intensity spatial (or angular) correlation function when the medium is illuminated by an internal source (or a source placed close to its surface), through the so-called $C_{0}$ contribution. This contribution equals the fluctuations of the local density of states (LDOS), another directly measurable quantity, that is also extremely sensitive to local microscopic scales due to near-field interactions. The scale-dependent morphology of the scattering medium also determines the maximum anisotropy length (MAL). This length scale characterizes the interaction between a complex medium and polarized radiation. Incorporating vectorial interaction length scales such as MAL into a transport equation would permit a consistent treatment of coherence and polarization transfer. Due to its relevance across many areas of research, the study of polarization-related phenomena in complex electromagnetic fields is a topic of intense current scrutiny.

\section{Acknowledgments}

This work was supported by LABEX WIFI (Laboratory of Excellence within the French Program "Investments for the Future") under references ANR-10-LABX-24 and ANR-10-IDEX-0001-02 PSL*.

\section{References}

[1] J.W. Goodman, Statistical Optics, Wiley, New York, 1985.

[2] L. Mandel, E. Wolf, Optical Coherence and Quantum Optics, Cambridge Univ. Press, Cambridge, 1995.

[3] E. Collett, Polarized Light Fundamentals and Applications, Marcel Dekker, New York, 1993.

[4] C. Brosseau, Fundamentals of Polarized Light a Statistical Optics Approach, Wiley, New York, 1998.

[5] E. Wolf, Introduction to the Theory of Coherence and Polarization, Cambridge Univ. Press, Cambridge, 2007.

[6] For a recent review of the most significant events in the evolution of polarization concepts, see C. Brosseau, A. Dogariu, Symmetry properties and polarization descriptors for an arbitrary electromagnetic wave filed, in: Progress in Optics, Elsevier, 2006, pp. 315-380.

[7] J.W. Goodman, Speckle Phenomena in Optics, Roberts and Company, Greenwood, 2007.

[8] B.J. Berne, R. Pecora, Dynamic Light Scattering, Wiley, New York, 1990.

[9] Second-order field correlations are sufficient to describe phenomena such as radiation of light, propagation, diffraction and interference. However, in general, higher-order correlations involving multiple products and powers of field variables are necessary to describe all the statistical properties of random electromagnetic fields. See for instance R.J. Glauber, Phys. Rev. 6 (1963) 2529.

[10] E. Wolf, Phys. Lett. A 312 (2003) 263.

[11] T. Setalä, J. Tervo, A.T. Friberg, Opt. Lett. 29 (2004) 328.

[12] P. Réfrégier, F. Goudail, Opt. Express 13 (2005) 6051.

[13] B.N. Simon, S. Simon, F. Gori, M. Santarsiero, R. Borghi, N. Mukunda, R. Simon, Phys. Rev. Lett. 104 (2010) 023901.

[14] Emil Wolf, Nuovo Cimento 12 (1954) 884.

[15] R.V. Langmuir, Appl. Phys. Lett. 2 (1963) 29.

[16] J. Ellis, A. Dogariu, J. Opt. Soc. Am. A 21 (2004) 988

[17] J. Ellis, A. Dogariu, J. Opt. Soc. Am. A 21 (2005) 491.

[18] I. Freund, M. Kaveh, R. Berkovits, M. Rosenbluh, Phys. Rev. B 42 (1990) R2613.

[19] S.M. Cohen, D. Eliyahu, I. Freund, M. Kaveh, Phys. Rev. A 43 (1991) R5748.

[20] J. Ellis, A. Dogariu, Opt. Lett. 29 (2004) 536.

[21] J. Broky, J. Ellis, A. Dogariu, Opt. Express 16 (2008) 14469.

[22] J. Broky, A. Dogariu, Opt. Express 18 (2010) 20105.

[23] R.K. Singh, D.N. Naik, H. Itou, Y. Miyamoto, M. Takeda, Opt. Express 19 (2011) 11558.

[24] E. Akkermans, G. Montambaux, Mesoscopic Physics of Electrons and Photons, Cambridge University Press, Cambridge, 2007.

[25] P. Sheng, Introduction to Wave Scattering, Localization, and Mesoscopic Phenomena, Academic Press, San Diego, 1995.

[26] S.M. Rytov, Yu.A. Kravtsov, V.I. Tatarskii, Principles of Statistical Radiophysics, vol. 4, Springer-Verlag, Berlin, 1989.

[27] L. Tsang, J.A. Kong, Scattering of Electromagnetic Waves: Advanced Topics, Wiley, New York, 2001.

[28] U. Frisch, in: A.A. Bharucha-Reid (Ed.), Probabilistic Methods in Applied Mathematics, vols. I and II, Academic, New York, 1968.

[29] The calculation of the effective dielectric function $\epsilon_{\text {eff }}$ based on the model of disorder with gaussian correlation function (Eq. (27)) can be adapted from that of chapter 4 in Ref. [27], in which the calculation is performed for a disorder with an exponential correlation function. A condition of validity of Eq. (30) is $6 \pi /\left(k_{0}^{4} \ell_{s} \ell_{c}^{3}\right) \ll \epsilon_{b}^{2}$, in addition to condition (28). 
[30] Defintions of intrinsic degrees of coherence and polarization can be introduced for 3D fields. See Ph. Réfrégier, V. Wasik, K. Vynck, R. Carminati, Opt. Lett. 39 (2014) 2362.

[31] This identity is valid for the Green function in a homogeneous medium in the presence of dissipation. Here the averaged Green function undergoes an effective dissipation due to attenuation by scattering.

[32] The dyadic Green function exhibits a non-integrable singularity at at $R=0$, that has to be treated with care [33]. Since the Dyson equation has been considered in the limit of large scale variations of the field compared to $\ell_{c}$, this singularity does not play any role in Eq. (40) since $\left|\mathbf{r}-\mathbf{r}^{\prime}\right|$ has to remain larger than $|\mathbf{X}| \simeq \ell_{c}$.

[33] J. van Bladel, Singular Electromagnetic Fields and Sources, Clarendon, Oxford, 1999.

[34] B. Shapiro, Phys. Rev. Lett. 57 (1986) 2168;

R. Pnini, B. Shapiro, Phys. Rev. B 39 (1989) 6986.

[35] S.M. Rytov, Yu.A. Kravtsov, V.I. Tatarskii, Principles of Statistical Radiophysics, vol. 3, Springer-Verlag, Berlin, 1989, (Chapter 3).

[36] L.D. Landau, E.M. Lifshitz, L.P. Pitaevskii, Statistical Physics, third ed., Pergamon Press, Oxford, 1980, Part 1, Chap. XII and Part 2, Chap. VIII.

[37] G.S. Agarwal, Phys. Rev. A 11 (1975) 230.

[38] K. Joulain, J.P. Mulet, F. Marquier, R. Carminati, J.-J. Greffet, Surf. Sci. Rep. 57 (2005) 59.

[39] R. Carminati, Phys. Rev. A 81 (2010) 053804.

[40] T. Setälä, K. Blomstedt, M. Kaivola, A.T. Friberg, Phys. Rev. E 67 (2003) 026613.

[41] R.L. Weaver, O.I. Lobkis, Phys. Rev. Lett. 87 (2001) 134301;

B.A. van Tiggelen, Phys. Rev. Lett. 91 (2003) 243904;

A. Derode, E. Larose, M. Tanter, J. de Rosny, A. Tourin, M. Campillo, M. Fink, J. Acoust, Soc. Am. 113 (2003) 2973;

M. Campillo, A. Paul, Science 299 (2003) 547;

M. Davy, M. Fink, J. de Rosny, Phys. Rev. Lett. 110 (2013) 203901.

[42] A. Cazé, R. Pierrat, R. Carminati, Phys. Rev. Lett. 110 (2013) 063903.

[43] K. Joulain, R. Carminati, J.P. Mulet, J.-J. Greffet, Phys. Rev. B 68 (2003) 245405.

[44] C. Sauvan, J.P. Hugonin, R. Carminati, P. Lalanne, Phys. Rev. A 89 (2014) 043825.

[45] J. de Rosny, M. Fink, Phys. Rev. Lett. 89 (2002) 124301.

[46] R. Carminati, R. Pierrat, J. de Rosny, M. Fink, Opt. Lett. 32 (2007) 3107.

[47] M.P. van Albada, A. Lagendijk, Phys. Rev. B 36 (1987) 2353.

[48] M. Rosenbluh, I. Edrei, M. Kaveh, I. Freund, Phys. Rev. A 35 (1987) 4458

[49] R. Vreeker, M. van Albada, R. Sprik, A. Lagendijk, Opt. Commun. 70 (1989) 365

[50] F.C. MacKintosh, J.X. Zhu, D.J. Pine, D.A. Weitz, Phys. Rev. B 40 (1989) 9342.

[51] A. Dogariu, C. Kutsche, P. Likamwa, G. Boreman, B. Moudgil, Opt. Lett. 22 (1997) 585.

[52] L.F. Rojas-Ochoa, D. Lacoste, R. Lenke, P. Schurtenberger, F. Scheffold, J. Opt. Soc. Am. A 21 (2004) 1799.

[53] M.J. Stephen, G. Cwilich, Phys. Rev. B 34 (1986) 7564.

[54] F.C. MacKintosh, S. John, Phys. Rev. B 37 (1988) 1884.

[55] V.D. Ozrin, Waves Random Media 2 (1992) 141.

[56] B.A. van Tiggelen, R. Maynard, T.M. Nieuwenhuizen, Phys. Rev. E 53 (1996) 2881.

[57] C.A. Müller, C. Miniatura, J. Phys. A: Math. Gen. 35 (2002) 10163.

[58] E. Akkermans, P.E. Wolf, R. Maynard, Phys. Rev. Lett. 56 (1986) 1471; J. Phys. 49 (1988) 77.

[59] A.S. Martinez, R. Maynard, Phys. Rev. B 50 (1994) 3714.

[60] M. Xu, R.R. Alfano, Phys. Rev. Lett. 95 (2005) 213901.

[61] E. Amic, J.M. Luck, T.M. Nieuwenhuizen, J. Phys. I (France) 7 (1997) 445.

[62] E.E. Gorodnichev, A.I. Kuzovlev, D.B. Rogozkin, J. Exp. Theor. Phys. 104 (2007) 319.

[63] K. Vynck, R. Pierrat, R. Carminati, Phys. Rev. A 89 (2014) 013842.

[64] S. Chandrasekhar, Radiative Transfer, Dover, New York, 1960.

[65] L.A. Apresyan, Y.A. Kravtsov, Radiation Transfer-Statistical and Wave Aspects, Gordon and Breach, Amsterdam, 1996.

[66] R. Pierrat, J.J. Greffet, R. Carminati, R. Elaloufi, J. Opt. Soc. Am. A 22 (2005) 2329.

[67] T. Setalä, M. Kaivola, A.T. Friberg, Phys. Rev. Lett. 88 (2002) 123902;

T. Setalä, A. Shevchenko, M. Kaivola, A.T. Friberg, Phys. Rev. E 66 (2002) 016615

[68] H. Roychowdhury, E. Wolf, Opt. Lett. 28 (2003) 170; J. Mod. Optics 51 (2004) 1603.

[69] This defintion of $\gamma_{E}\left(\mathbf{r}, \mathbf{r}^{\prime}\right)$ differs from that in Ref. [39] in which no normalization was used.

[70] J.E. Sipe, J. Opt. Soc. Amer. B Opt. Phys. 4 (1987) 481.

[71] Compared to the expression given in Ref. [39], a misprint has been corrected. The factor $1 / \gamma_{1}$ in the integral is the correct one.

[72] The amplitude transmission factors are $t_{s}=2 \gamma_{1} /\left(\gamma_{0}+\gamma_{1}\right)$ and $t_{p}=2 \sqrt{\epsilon_{\text {eff }}} \gamma_{1} /\left(\epsilon_{\text {eff }} \gamma_{0}+\gamma_{1}\right)$. The amplitude reflection factors are $r_{s}=$ $\left(\gamma_{0}-\gamma_{1}\right) /\left(\gamma_{0}+\gamma_{1}\right)$ and $r_{p}=\left(\epsilon_{\text {eff }} \gamma_{0}-\gamma_{1}\right) /\left(\epsilon_{\text {eff }} \gamma_{0}+\gamma_{1}\right)$.

[73] J.-J. Greffet, R. Carminati, Prog. Surf. Sci. 56 (1997) 133.

[74] L. Novotny, B. Hecht, Principles of Nano-Optics, Cambridge University Press, Cambridge, 2006.

[75] I. Freund, D. Eliyahu, Phys. Rev. A 45 (1992) 6133.

[76] See E. Gutiérrez-Reyes, A. García-Valenzuela, R.G. Barrera, J. Phys. Chem. B 118 (2014) 6015. and references therein.

[77] R. Carminati, J.-J. Greffet, Phys. Rev. Lett. 82 (1999) 1660.

[78] C. Henkel, K. Joulain, R. Carminati, J.-J. Greffet, Opt. Commun. 186 (2000) 57.

[79] C. Henkel, K. Joulain, Appl. Phys. B 84 (2006) 61.

[80] J.-J. Greffet, R. Carminati, Ultramicroscopy 61 (1995) 43.

[81] J.-J. Greffet, R. Carminati, Speckle pattern in the near field, in: A.A. Maradudin (Ed.), Light Scattering and Nanoscale Surface Roughness, in: Springer Series on Nanostructure Science and Technology, Springer, New York, 2007, chapter 15.

[82] A. Apostol, A. Dogariu, Phys. Rev. Lett. 91 (2003) 093901.

[83] A. Apostol, A. Dogariu, Opt. Lett. 29 (2004) 235.

[84] A. Apostol, A. Dogariu, Phys. Rev. E 72 (2005) 025602(R).

[85] V. Emiliani, F. Intonti, M. Cazayous, D.S. Wiersma, M. Colocci, F. Aliev, A. Lagendijk, Phys. Rev. Lett. 90 (2003) 250801.

[86] V.M. Apalkov, M.E. Raikh, B. Shapiro, Phys. Rev. Lett. 92 (2004) 253902.

[87] V.M. Shalaev, Nonlinear Optics of Random Media, Springer, Berlin, 2000

[88] K. Seal, A.K. Sarychev, H. Noh, D.A. Genov, A. Yamilov, V.M. Shalaev, Z.C. Ying, H. Cao, Phys. Rev. Lett. 94 (2005) 226101.

[89] J. Laverdant, S. Buil, B. Bérini, X. Quélin, Phys. Rev. B 77 (2008) 165406.

[90] B. Shapiro, Phys. Rev. Lett. 83 (1999) 4733.

[91] S. Feng, C. Kane, P.A. Lee, A.D. Stone, Phys. Rev. Lett. 61 (1988) 834.

[92] S.E. Skipetrov, R. Maynard, Phys. Rev. B 62 (2000) 886.

[93] A. Cazé, R. Pierrat, R. Carminati, Phys. Rev. A 82 (2010) 043823.

[94] R. Sapienza, P. Bondareff, R. Pierrat, B. Habert, R. Carminati, N.F. van Hulst, Phys. Rev. Lett. 106 (2011) 163902.

[95] B.A. van Tiggelen, S.E. Skipetrov, Phys. Rev. E 73 (2006) 045601(R).

[96] J.D. Jackson, Classical Electrodynamics, Wiley, New York, 1962, Chapter 3, section 3.5 
[97] The factorization of the intensity-intensity correlation function for Gaussian fields is given by Eq. (14) in T. Setälä, K. Lindfors, M. Kaivola, J. Tervo, A.T. Friberg, Opt. Lett. 29 (2004) 2587. Only the trace of the cross-sepctral density matrix contributes since different components of the field are uncorrelated after a few mean free paths, according to the calculation in section 2.3.7.

[98] The LDOS $\rho(\mathbf{r}, \omega)=\left[2 \omega /\left(\pi c^{2}\right)\right] \operatorname{Im}[\operatorname{Tr} \mathbf{G}(\mathbf{r}, \mathbf{r}, \omega)]$ is actually the electric contribution to the full LDOS, that enters the expression of the spontaneous decay rate of an electric dipole. For magnetic dipole transitions, or for the computation of equilibrium energy density, an additional magnetic term has to be added, see [43].

[99] R.A.L. Vallée, M. Van der Auweraer, W. Paul, K. Binder, Phys. Rev. Lett. 97 (2006) 217801.

[100] P.V. Ruijgrok, R. Wüest, A.A. Rebane, A. Renn, V. Sandoghdar, Opt. Express 18 (2010) 6360.

[101] M.D. Birowosuto, S.E. Skipetrov, W.L. Vos, A.P. Mosk, Phys. Rev. Lett. 105 (2010) 013904

[102] V. Krachmalnicoff, E. Castanié, Y. De Wilde, R. Carminati, Phys. Rev. Lett. 105 (2010) 183901.

[103] E. Castanié, V. Krachmalnicoff, A. Cazé, R. Pierrat, Y. De Wilde, R. Carminati, Opt. Lett. 37 (2012) 3006.

[104] A.D. Mirlin, Phys. Rep. 326 (2000) 259.

[105] H. Schomerus, M. Titov, P.W. Brouwer, C.W.J. Beenakker, Phys. Rev. B 65 (2002) 121101.

[106] P.D. García, R. Sapienza, L.S. Froufe-Pérez, C. López, Phys. Rev. B 79 (2009) 241109.

[107] R. Pierrat, R. Carminati, Phys. Rev. A 81 (2010) 063802.

[108] L.S. Froufe-Pérez, R. Carminati, J.J. Sáenz, Phys. Rev. A 76 (2007) 013835.

[109] L.S. Froufe-Pérez, R. Carminati, Phys. Status Solidi (a) 205 (2008) 1258.

[110] R. Carminati, J.-J. Greffet, C. Henkel, J.M. Vigoureux, Opt. Commun. 261 (2006) 368.

[111] S. Albaladejo, R. Gómez-Medina, L. Froufe-Pérez, H. Marinchio, R. Carminati, G. Armelles, J. Torrado, A. García-Martín, J. Sáenz, Opt. Express 18 (2010) 3556.

[112] The decay rate $\Gamma$ of a quantum electric-dipole emitter placed at position $\mathbf{r}_{s}$ and emitting at frequency $\omega$ is proportional to the LDOS $\rho\left(\mathbf{r}_{s}, \omega\right)$. Therefore the expressions of the averaged value and variance of $\rho / \rho_{0}$ can be identified with that of $\Gamma / \Gamma_{0}$ given in Refs. [108,109].

[113] A. Apostol, D. Haefner, A. Dogariu, Phys. Rev. E 74 (2006) 066603.

[114] D. Haefner, S. Sukhov, A. Dogariu, Phys. Rev. E 81 (2010) 016609.

[115] D. Haefner, S. Sukhov, A. Dogariu, Phys. Rev. Lett. 100 (2008) 04390

[116] S. Sukhov, D. Haefner, A. Dogariu, J. Opt. Soc. Am. A 27 (2010) 827.

[117] L. Tsang, J.A. Kong, K.H. Ding, Scattering of ElectromagneticWaves: Numerical Simulations, Wiley, New York, 2001.

[118] B.T. Draine, P.J. Flatau, J. Opt. Soc. Am. A 11 (1994) 1491.

[119] K.D. Bonin, V.V. Kresin, Electric-Dipole Polarizabilities of Atoms, Molecules and Clusters, World Scientific, Singapore, 1997.

[120] S.V. Karpov, V.S. Gerasimov, I.L. Isaev, V.A. Markel, Phys. Rev. B 72 (2005) 205425. 